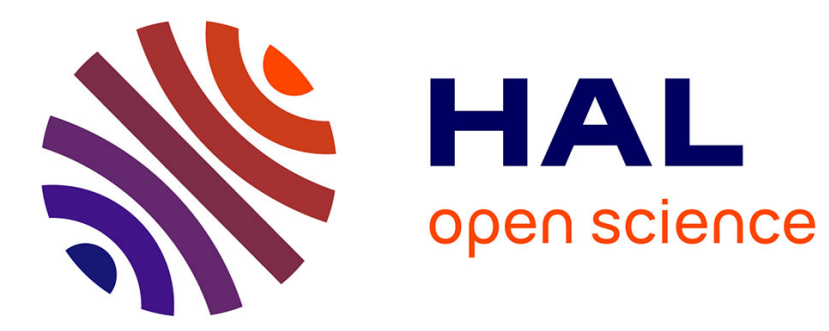

\title{
Cones as a model of intuitionistic linear logic
}

Thomas Ehrhard

\section{To cite this version:}

Thomas Ehrhard. Cones as a model of intuitionistic linear logic. LICS '20: 35th Annual ACM/IEEE Symposium on Logic in Computer Science, Jul 2020, Saarbrücken Germany, Germany. pp.370-383, 10.1145/3373718.3394758 . hal-02436161v2

\section{HAL Id: hal-02436161 \\ https://hal.science/hal-02436161v2}

Submitted on 14 Dec 2020 (v2), last revised 17 Oct 2021 (v3)

HAL is a multi-disciplinary open access archive for the deposit and dissemination of scientific research documents, whether they are published or not. The documents may come from teaching and research institutions in France or abroad, or from public or private research centers.
L'archive ouverte pluridisciplinaire HAL, est destinée au dépôt et à la diffusion de documents scientifiques de niveau recherche, publiés ou non, émanant des établissements d'enseignement et de recherche français ou étrangers, des laboratoires publics ou privés. 


\title{
Cones as a model of intuitionistic linear logic
}

\author{
Thomas Ehrhard \\ ehrhard@irif.fr \\ Université de Paris, IRIF, CNRS, F-75013 Paris, France
}

\begin{abstract}
For overcoming the limitations of probabilistic coherence spaces which do not seem to provide natural interpretations of continuous data types such as the real line, we introduced with Pagani and Tasson a model of probabilistic higher order computation based on (positive) cones, and a class of totally monotone functions that we called "stable". Then Crubille proved that this model is a conservative extension of the earlier probabilistic coherence space model. We continue these investigations by showing that the category of cones and linear and Scott-continuous functions is a model of intuitionistic linear logic. To define the tensor product, we use the special adjoint functor theorem, and we prove that this operation is an extension of the standard tensor product of probabilistic coherence spaces. We also show that these latter are dense in cones, thus allowing to lift the main properties of the tensor product of probabilistic coherence spaces to general cones. Finally we define in the same way an exponential of cones and extend measurability to these new operations.
\end{abstract}

Keywords: Denotational semantics, probabilistic programming languages, linear logic

\section{Introduction}

We continue a series of investigations initiated in [4] on a class of models of higher order computation, based on an initial idea of Girard [11]. In these models, types are interpreted as concrete structures called probabilistic coherence spaces (PCSs) consisting of a set (the web) and a collection of $\mathbb{R}_{\geq 0}$-valued families indexed by the web generalizing discrete probability distributions: a typical example of PCS is $\mathbb{N}$ equipped with subprobability ${ }^{1}$ distributions on $\mathbb{N}$. Another example is $\mathbb{N} \times \mathbb{N}$ equipped with all families $\left(t_{i, j}\right)_{(i, j) \in \mathbb{N} \times \mathbb{N}}$ such that, for all subprobability distribution $\left(x_{i}\right)_{i \in \mathbb{N}}$ on $\mathbb{N}$, the family $\left(\sum_{i \in \mathbb{N}} t_{i, j} x_{i}\right)_{j \in \mathbb{N}}$ is a subprobability distribution on $\mathbb{N}$. Such a $t$ is a $\mathbb{N} \times \mathbb{N}$ substochastic matrix which represents a sub-Markov process with $\omega$ states. In [4] it is proven that PCSs are a categorical model of classical linear logic (LL), that is, a Seely category $\left([15]^{2}\right)$ Pcoh, where all recursive types can be interpreted, and which provides an adequate interpretation of a probabilistic extension of Plotkin's PCF [16].

\footnotetext{
${ }^{1}$ Not probability, in order to interpret also partial computation.

${ }^{2}$ Our main reference for the category theory of models of linear logic, see also that paper for thorough discussions on the complicated history of the notions involved.
}

$[2,6-8,10]$ extended these results, proving full abstraction properties for probabilistic versions of PCF and Levy's Call-by-Push-Value, and proving that the exponential of PCSs introduced in [4] is the free one. One essential feature of this model is that the morphisms of the associated Kleisli category are extremely regular and can be seen as analytic functions, some consequences of this fact are presented in in [5] and crucially used in proofs of full abstraction.

The main weakness of the PCS model is that it does not host "continuous data types" such as the real line equipped with its standard Borel $\Sigma$-algebra, required for taking into account modern probabilistic languages used in Bayesian programming. [4] suggested that PCSs might be generalized using a well-suited notion of ordered Banach space or positive cone. This was done in [9], using a notion of positive cone $^{3}$ considered earlier in particular in [17]. Any PCS gives rise naturally to such a cone, and one can also associate with any measurable space the cone of all measures which have a finite global weight. [9] shows that, equipped with suitable stable morphisms (which are Scott-continuous functions satisfying a total monotonicity requirement which has some similarities with Berry's stability), these objects form a cartesian closed category (CCC) Cstab providing an adequate interpretation of an extension of PCF with a type of real numbers and a sampling primitive. Then, Crubille showed that this CCC contains the Kleisli category of the PCS model as a full sub-CCC [1], providing a very satisfactory connection between these constructions.

Following [17], it is noticed in [9] that there is a natural notion of linear and Scott-continuous functions between cones, which coincides with the notion of linear morphisms of Pcoh when restricted to cones induced by PCSs: this defines the category CLin we study here. Given cones $P$ and $Q$, one can build a cone $P \multimap Q$ whose elements are those of $\operatorname{CLin}(P, Q)$ so we could reasonably expect the functor $P \multimap \ldots$ to have a left adjoint for each $P$, hopefully turning CLin into a symmetric monoidal closed category (SMCC) ${ }^{4}$.

With cones $P$ and $Q$ we should associate functorially a cone $P \otimes Q$ such that (at least) there is a natural bijection between $\operatorname{CLin}(P \otimes Q, R)$ and $\operatorname{CLin}(P, Q \multimap R)$. Our first attempt was concrete: since the elements of this second homset are continuous and bilinear functions $P \times Q \rightarrow R$, our tensor product should classify such functions and hence it was

\footnotetext{
${ }^{3}$ There is a long tradition of research on this kind of structures, rooted in the theory of Banach spaces. Such cones have been used in semantics quite successfully for instance in [13] and subsequent work.

${ }^{4}$ Probably not a *autonomous category however.
} 
natural to look for $P \otimes Q$ as a sub-cone ${ }^{5}$ of $\mathcal{B}(P, Q)^{\prime}$ where $\mathcal{B}(P, Q)$ is the cone of continuous bilinear maps $P \times Q \rightarrow$ $\mathbb{R}_{\geq 0}$ and $R^{\prime}=\left(R \multimap \mathbb{R}_{\geq 0}\right)$ (the dual of $R$ ): with any $x \in P$ and $y \in Q$ we can indeed associate the linear and continuous function $x \otimes y: \mathcal{B}(P, Q) \rightarrow \mathbb{R}_{\geq 0}, f \mapsto f(x, y)$. Whence a definition of $P \otimes Q$ : the least subcone of $\mathcal{B}(P, Q)^{\prime}$ which contains all the $x \otimes y$, for $x \in P$ and $y \in Q$. This also gives us a continuous and bilinear map $\tau: P \times Q \rightarrow P \otimes Q$, $(x, y) \mapsto x \otimes y$.

We should now prove the universal property: for any bilinear and continuous $f: P \times Q \rightarrow R$, there is exactly one $\widehat{f} \in \operatorname{CLin}(P \otimes Q, R)$ such that $f=\widehat{f} \tau$. It is easy to define $\widehat{f}$ on the elements of $P \otimes Q$ of shape $x \otimes y$ (under a mild separateness assumption on our cones), but how can we extend this map to the whole of $P \otimes Q$ ? Our "top-down" definition of $P \otimes Q$ is ineffective for this, we need a "bottom-up" approach, something like: an element of $P \otimes Q$ is a (possibly infinite) linear combination $\sum_{i \in I} \alpha_{i}(x(i) \otimes y(i))$ where $\alpha_{i} \in \mathbb{R}_{\geq 0}$ and $\sum_{i \in I} \alpha_{i}=1$ (convex combination of pure tensors). But this is not enough because we could perfectly have two convex combinations of pure tensors $z$ and $z^{\prime}$ such that $z^{\prime} \leq z$ (in $\left.\mathcal{B}(P, Q)^{\prime}\right)$ and then $P \otimes Q$ will also contain $z-z^{\prime}$ (Example 13 shows that such subtractions are mandatory at least if we want our $\otimes$ to extend that of Pcoh). In the usual algebraic case, coefficients form a ring and such elements are just combinations of pure tensors, with possibly negative coefficients. Here on the contrary we have to take such differences into account explicitly since our coefficients are in $\mathbb{R}_{\geq 0}$.

Another problem arises from the very peculiar completeness of cones and continuity of morphisms, which are defined purely in terms of the algebraic order relation (according to which $x_{1} \leq x_{2}$ if there exists $x$ such that $x_{1}+x=x_{2}$ ), and not of the norm: if a given element $z$ of $P \otimes Q$ can be written in two different ways as a convex combination of pure tensors $z=\sum_{i \in I} \alpha_{i}(x(i) \otimes y(i))=\sum_{j \in J} \beta_{j}\left(x^{\prime}(j) \otimes y^{\prime}(j)\right)$, it is not obvious, though certainly true, that $\sum_{i \in I} \alpha_{i} f(x(i), y(i))=$ $\sum_{j \in J} \beta_{j} f\left(x^{\prime}(j), y^{\prime}(j)\right)$.

Contents. After several attempts, we arrived to the conclusion that the concrete approach would lead to rather complicated (though quite interesting) developments. Fortunately a shorter road was open, based on the following observation: our category CLin is small complete and the functor $P \multimap$ preserves all small limits so we are in position of applying the special adjoint functor theorem (because CLin is also well-powered, and, under the aforementioned separateness condition on objects, it admits $\mathbb{R}_{\geq 0}$ as cogenerating object). So the functor $P \multimap \ldots$ has a left adjoint: we get our tensor product $\otimes$ almost for free! This is not the end of the story however because the simple fact that $\otimes$ is a bifunctor defined as a left adjoint to $\multimap$ is not sufficient to prove that

\footnotetext{
${ }^{5}$ A notion to be defined carefully.
}

it defines a monoidal structure. Though, we are lucky again because

- it turns out that Pcoh is a dense subcategory of CLin (that is, any cone is a colimit of a diagram of PCSs), which per se is quite an interesting property;

- being a left adjoint, $\otimes$ commutes with all existing colimits of cones;

- restricted to Pcoh, our new tensor product coincides with the ordinary one, which defines a monoidal structure on Pcoh.

Combining these facts we lift the monoidal structure (associativity isomorphisms etc) from Pcoh to CLin, thus proving that CLin is an SMCC, which contains Pcoh as a full sub-SMCC and this was our main goal.

Then we use the same method to define an exponential functor ! : CLin $\rightarrow$ CLin and show that it is a resource modality in the sense of Seely categories (again, see [15]).

We conclude the paper by explaining shortly how the measurability structure introduced for cones in [9] can be extended to our tensor product and exponential. Such structures are indeed essential for interpreting the sampling constructs of probabilistic programming languages.

Related work. Positive cones have been used in various contexts in the semantics of probabilistic programming languages, notably under the name of Kegelspitzen (which are "unit balls" of cones) for which we refer to $[12,13]$. The main difference with our approach is that such cones are usually equipped with an additional "extensional" order relation whereas the only order relation we consider in our work is the algebraic one: this constraint, strongly suggested by PCSs, obliged the authors of [9] to introduce stable functions.

Closer to our approach are [18] and [3] where types are interpreted as ordered Banach spaces and tensor products are also defined. The main difference that we can see between their approaches and ours is that they put more standard continuity requirements on linear morphisms, based on the norm, whereas we insist on our linear (and stable) morphisms to be Scott continuous, a purely ${ }^{6}$ order-theoretic notion which implies boundedness and thus norm-based continuity, but the converse implication does not hold in general. The main benefit of insisting on this kind of continuity is that, our stable morphisms being Scott-continuous, they have least fixed points (and by cartesian closeness, the function computing these fixed points is itself stable). Deeply related with this choice is the fact that stable functions are defined only on the unit ball of the source cone: the use of fixed points prevents in general stable functions from being extended to the whole cone, see [5] for examples illustrating this fact.

\footnotetext{
${ }^{6}$ Not completely actually, since we require commutation with lubs of bounded monotone sequences, and the definition of boundedness involves the norm.
} 


\section{Density}

The categorical notion of density (see [14], Chap. X Sec. 6) plays a crucial role, we spend some time to introduce it and present useful properties ${ }^{7}$. But we start with the following simple lemma which will be quite useful.

Lemma 1. Let $\mathrm{C}$ and $\mathrm{D}$ be categories, $F, G: \mathrm{C} \rightarrow \mathrm{D}$ be functors and $\psi_{C, D}: \mathbf{D}(F(C), D) \rightarrow \mathbf{D}(G(C), D)$ be a natural bijection. Then the family of morphisms $\eta_{C}=\psi_{C, F(C)}\left(\operatorname{Id}_{F(C)}\right) \in$ $\mathrm{D}(G(C), F(C))$ is a natural isomorphism whose inverse is the family of morphisms $\theta_{C}=\psi_{C, G(C)}^{-1}\left(\operatorname{Id}_{G(C)}\right) \in \mathbf{D}(F(C), G(C))$.

A functor $F: \mathrm{C} \rightarrow \mathrm{D}$ is cocontinuous if it preserves all small colimits which exist in $\mathrm{C}$ : given a functor $\Delta: J \rightarrow \mathrm{C}$ where $J$ is small (one says that $\Delta$ is a diagram) and given a colimiting cocone $\gamma: \Delta \Rightarrow c$ on $\Delta$ (initial object in the category of cocones on $\Delta$ ) for some object $c$ of $\mathrm{C}$, then the cocone $F \gamma: F \Delta \Rightarrow F(c)$ is a colimiting cocone in D.

Given categories D and E, we use [D, E] for the category of functors and natural transformations from $\mathrm{D}$ to $\mathrm{E}$.

Lemma 2. Let $F: \mathrm{C} \times \mathrm{D} \rightarrow \mathrm{E}$ be a functor which is cocontinuous in its first argument (that is, given any object $d$ of $\mathrm{D}$, the functor $F(\ldots, d)$ is cocontinuous). Then the curried functor $F^{\prime}: \mathrm{C} \rightarrow[\mathrm{D}, \mathrm{E}]$ is cocontinuous.

Let $I: \mathrm{C}^{0} \rightarrow \mathrm{C}$ (where we assume $\mathrm{C}^{0}$ to be small) and let $c \in \operatorname{Obj}(\mathrm{C})$. Let $I / c$ be the comma category (its objects are the pairs $(x, f)$ where $x \in \operatorname{Obj}\left(\mathrm{C}^{0}\right)$ and $f \in \mathrm{C}(I(x), c)$ and $I / c((x, f),(y, g))$ is the set of all $t \in \mathbf{C}^{0}(x, y)$ such that $g I(t)=f)$ and $\Delta_{c}: I / c \rightarrow \mathrm{C}$ be the functor which maps $(x, f)$ to $I(x)$ and similarly for morphisms. Let $\gamma^{c}: \Delta_{c} \Rightarrow c$ be the cocone defined by $\gamma_{(x, f)}^{c}=f$. One says that the functor $I$ is dense (see [14], Chap. X, Sec. 6) if $\gamma^{c}$ is a colimiting cocone for each $c \in \operatorname{Obj}(C)$. If $\mathrm{C}^{0}$ is a full subcategory of $\mathrm{C}$ and $I$ is the inclusion, $\mathrm{C}^{0}$ is said to be a dense subcategory of $\mathrm{C}$.

Lemma 3. Let $I: \mathrm{C}^{0} \rightarrow \mathrm{C}$ be dense and let $F: \mathrm{C} \rightarrow \mathrm{D}$ be cocontinuous. Let $c \in \operatorname{Obj}(\mathrm{C}), d \in \mathrm{Obj}(\mathrm{D})$ and $l, l^{\prime} \in$ $\mathrm{D}(F(c), d)$. If, for all $x \in \mathrm{C}^{0}$ and $f \in \mathrm{C}(I(x), c)$ one has $l F(f)=l^{\prime} F(f)$ then $l=l^{\prime}$.

Proof. By our assumption on $l$ and $l^{\prime}$ we define in $\mathrm{D}$ a unique cocone $\delta: F \Delta_{c} \Rightarrow d$ by setting $\delta_{(x, f)}=l F(f)=l^{\prime} F(f)$ and the fact that $F \gamma^{c}$ is a colimiting cocone (because $F$ is cocontinuous) implies that $l=l^{\prime}$.

Lemma 4. Let $I: \mathrm{C}^{0} \rightarrow \mathrm{C}$ be dense, let $F, G: \mathrm{C} \rightarrow \mathrm{D}$ be functors and assume that $F$ is cocontinuous. Let $\tau: F I \Rightarrow G I$, there is exactly one $\widetilde{\tau}: F \Rightarrow G$ such that $\widetilde{\tau} I=\tau$. Moreover if $\tau$ is a natural isomorphism and $G$ is also cocontinuous, then $\widetilde{\tau}$ is an isomorphism.

\footnotetext{
${ }^{7}$ There is no doubt that they are all quite standard, we provide the statements in a form convenient for our purpose.
}

Now we extend the previous results to separately cocontinuous multi-ary functors since we want to apply them to our tensor product.

Lemma 5. For $i=1, \ldots, n$ let $I_{i}: \mathrm{C}_{i}^{0} \rightarrow \mathrm{C}_{i}$ be dense functors. Let $F: \prod_{i=1}^{n} \mathrm{C}_{i} \rightarrow \mathrm{D}$ be separately cocontinuous (that is, for each $i=1, \ldots, n$ and each $c_{1} \in \operatorname{Obj}\left(\mathrm{C}_{1}\right), \ldots, c_{i-1} \in$ $\operatorname{Obj}\left(\mathrm{C}_{i-1}\right), c_{i+1} \in \operatorname{Obj}\left(\mathrm{C}_{i+1}\right), \ldots, c_{n} \in \operatorname{Obj}\left(\mathbf{C}_{n}\right)$, the functor $F\left(c_{1}, \ldots, c_{i-1},{ }_{-}, c_{i+1}, \ldots, c_{n}\right)$ is cocontinuous). Let $\vec{c} \in \operatorname{Obj}\left(\prod_{i=1}^{n} \mathbf{C}_{i}\right)$, $d \in \operatorname{Obj}(D)$ and let $l, l^{\prime} \in \mathbf{D}(F(\vec{c}), d)$. If, for all $\vec{x} \in \operatorname{Obj}\left(\prod_{i=1}^{n} C_{i}^{0}\right)$ and all $\vec{f} \in \prod_{i=1}^{n} \mathrm{C}_{i}\left(I_{i}\left(x_{i}\right), c_{i}\right)$ one has $l F(\vec{f})=l^{\prime} F(\vec{f})$, then $l=l^{\prime}$.

Theorem 6. For $i=1, \ldots, n$ let $I_{i}: \mathrm{C}_{i}^{0} \rightarrow \mathrm{C}_{i}$ be dense functors. Let $F, G: \prod_{i=1}^{n} \mathrm{C}_{i} \rightarrow \mathrm{D}$ be functors and assume that $F$ is separately cocontinuous. For any natural transformation $\tau: F\left(\prod_{i=1}^{n} I_{i}\right) \Rightarrow G\left(\prod_{i=1}^{n} I_{i}\right)$, there is exactly one natural transformation $\tilde{\tau}: F \Rightarrow G$ such that $\tilde{\tau}\left(\prod_{i=1}^{n} I_{i}\right)=\tau$. If $G$ is also separately cocontinuous and if $\tau$ is a natural bijection, then $\tilde{\tau}$ is also a natural bijection.

\section{The category of cones and linear maps}

There is an unfortunate though hardly avoidable clash of terminology between the cones that we introduce now and the categorical cocones used to deal with density. We use "cone" for the former and "cocone" for the latter to prevent any misunderstanding.

A positive cone is a structure $\left(P,\left\|_{-}\right\|\right)$where $P$ is an $\mathbb{R}_{\geq 0^{-}}$ semimodule and $\left\|_{-}\right\|$is a function $P \rightarrow \mathbb{R}_{\geq 0}$ which satisfies the usual conditions of a norm ${ }^{8}$. It is assumed moreover that $P$ is cancellative (meaning $x_{1}+x=x_{2}+x \Rightarrow x_{1}=x_{2}$ ) and that $\left(P,\left\|_{-}\right\|\right)$is positive (meaning $\left.\left\|x_{1}\right\| \leq\left\|x_{1}+x_{2}\right\|\right)$. A subset $C$ of $P$ is bounded if $\{\|x\| \mid x \in C\}$ is bounded in $\mathbb{R}_{\geq 0}$. We use $\mathcal{B P}$ for the closed unit "ball" $\{x \in P \mid\|x\| \leq 1\}$. The algebraic order relation of $P$ is defined by: $x_{1} \leq x_{2}$ if $\exists x \in P x_{1}+x=x_{2}$. When such an $x$ exists it is unique by cancellativity, and we use the notation $x=x_{2}-x_{1}$; apart from its partiality, this subtraction obeys all the usual algebraic laws. One says that $P$ is complete if any monotone $\omega$-indexed ${ }^{9}$ sequence in $\mathcal{B P}$ has a lub which lies in $\mathcal{B P}$.

The semiring $\mathbb{R}_{\geq 0}$ is a complete positive cone, with norm defined as the identity.

Example 7. Given a measurable space $\mathcal{X}$ (with $\sum$-algebra $\left.\Sigma_{\mathcal{X}}\right)$, the set $\underline{\mathrm{M}}(\mathcal{X})$ of all non-negative measures $\mu$ on $\mathcal{X}$ such that $\mu(X)<\infty$ is a complete positive cone, when equipped with algebraic operations defined pointwise and norm $\|\mu\|=$ $\mu(\mathcal{X})$.

Continuity and linearity. When dealing with cones, the word "continuity" always applies to functions which are monotone wrt. the algebraic order, and means commutation with

\footnotetext{
${ }^{8}$ It is essential to notice that this norm is part of the structure of the cone. ${ }^{9}$ And not arbitrary directed sets as a domain-theorist might expect, because we need to apply the monotone convergence theorem of measure theory at some point.
} 
lubs of such monotone sequences in the unit ball. It is easy to check that all the operations of a cone (addition, scalar multiplication and norm) are monotone and continuous.

Given $P$ and $Q$ complete positive cones, a map $f: P \rightarrow Q$ is linear if it commutes with the algebraic operations. If $f$ is moreover continuous then it is not hard to prove that it is bounded in the sense that it maps $\mathcal{B P}$ to a bounded subset of $Q([17])$. Therefore we can define $\|f\|=\sup _{x \in \mathcal{B} P}\|f(x)\| \in$ $\mathbb{R}_{\geq 0}$. We use $P^{\prime}$ for the set of linear and continuous maps $P \rightarrow \mathbb{R}_{\geq 0}$. We say that $P$ is separated ${ }^{10}$ if $\left(\forall x^{\prime} \in P^{\prime}\left\langle x_{1}, x^{\prime}\right\rangle=\right.$ $\left.\left\langle x_{2}, x^{\prime}\right\rangle\right) \Rightarrow x_{1}=x_{2}$, where we set $\left\langle x, x^{\prime}\right\rangle=x^{\prime}(x)$ for $x \in P$ and $x^{\prime} \in P^{\prime}$.

Infinite sums. Let $P$ be a cone and $\vec{x}=\left(x_{i}\right)_{i \in I}$ be a family of elements of $P$ indexed by a set $I$ which is at most countable. We say that $\vec{x}$ is summable if the family of real numbers $\left(\left\|\sum_{i \in J} x_{i}\right\|\right)_{J \in \mathcal{P}_{\text {fin }}(I)}$ is bounded. In that case one can define $\sum_{i \in I} x_{i} \in P$ in an unique way. Indeed, take a monotone sequence $I(n)$ of finite subsets of $I$ such that $\bigcup_{n \in \mathbb{N}} I(n)=I$, then the sequence $\left(\sum_{i \in I(n)} x_{i}\right)_{n \in \mathbb{N}}$ is monotone and normbounded and hence has a lub in $P$. This lub does not depend on the choice of the sequence $(I(n))_{n \in \mathbb{N}}$ because any two such sequences are cofinal. We use $\sum_{i \in I} x_{i}$ for this lub. Obviously any sub-family of a summable family is summable.

Lemma 8. Let $f: P \rightarrow Q$ be linear and continuous. Then for any summable family $\left(x_{i}\right)_{i \in I}$ in $P$, the family $\left(f\left(x_{i}\right)\right)_{i \in I}$ is summable in $Q$ and we have $f\left(\sum_{i \in I} x_{i}\right)=\sum_{i \in I} f\left(x_{i}\right)$.

Lemma 9. Let $\vec{x}=\left(x_{i, j}\right)_{(i, j) \in I \times J}$ be a doubly-indexed family of elements of a cone $P$ and assume that for each $i \in I$ the family $\left(x_{i, j}\right)_{j \in J}$ is summable and that the family $\left(\sum_{j \in J} x_{i, j}\right)_{i \in I}$ is summable. Then the family $\vec{x}$ is summable and $\sum_{i \in I, j \in J} x_{i, j}=$ $\sum_{i \in I} \sum_{j \in J} x_{i, j}=\sum_{j \in J} \sum_{i \in I} x_{i, j}$.

We use CLin for the category whose objects are the separated complete positive cones and morphisms are the continuous linear functions whose norm is $\leq 1$, in other words, the $f: P \rightarrow Q$ which are linear and continuous and satisfy $f(\mathcal{B P}) \subseteq \mathcal{B Q}$.

\subsection{Linear function spaces}

Let $P$ and $Q$ be object of CLin, we define the cone $P \multimap$ $Q$ whose elements are the linear and continuous functions $P \rightarrow Q$ with algebraic operations defined pointwise and norm defined by $\|f\|=\sup _{x \in \mathcal{B} P}\|f(x)\|_{Q}$ which is welldefined by continuity of $f$. Notice that in this cone, the algebraic order relation coincides with the pointwise order on functions. Let indeed $f, g \in P \multimap Q$ be such that $\forall x \in$ $P f(x) \leq g(x)$. Then we define a linear function $h: P \rightarrow$ $Q$ by setting $h(x)=g(x)-f(x)$ by the usual laws satisfied by subtraction. Let us prove that this linear function $h$ is continuous so let $\left(x_{n}\right)_{n \in \mathbb{N}}$ be a non-decreasing sequence

\footnotetext{
${ }^{10}$ It is not completely clear to us that all cones are separated as one would expect with Banach spaces in mind.
}

in $\mathcal{B P}$ and let $x \in \mathcal{B P}$ be its lub, we need to prove that $h(x) \leq \sup _{n \in \mathbb{N}} h\left(x_{n}\right)$, the converse resulting from the monotonicity of $h$, that is, we have to prove that $g(x) \leq f(x)+$ $\sup _{n \in \mathbb{N}} h\left(x_{n}\right)$. Let $k \in \mathbb{N}$, one has $g\left(x_{k}\right)=f\left(x_{k}\right)+h\left(x_{k}\right) \leq$ $f(x)+\sup _{n \in \mathbb{N}} h\left(x_{n}\right)$ and we are done since $g$ is continuous.

The cone $P \multimap Q$ is complete, lubs being computed pointwise (since the order relation is the pointwise order on functions). This cone is separated because, given $f_{1}, f_{2} \in P \multimap Q$ with $f_{1} \neq f_{2}$ there exists $x \in P$ such that $f_{1}(x) \neq f_{2}(x)$ and hence there exists $y^{\prime} \in Q^{\prime}$ which separates $f_{1}(x)$ from $f_{2}(x)$. Now the operation $h \mapsto\left\langle h(x), y^{\prime}\right\rangle$ is an element of $(P \multimap Q)^{\prime}$ which separates $f_{1}$ from $f_{2}$.

Moreover the operation _ $\multimap \ldots$ is a functor CLin ${ }^{\text {op }} \times$ CLin $\rightarrow$ CLin, the action of morphisms being defined as follows. Let $f \in \operatorname{CLin}\left(P_{2}, P_{1}\right)$ and $g \in \operatorname{CLin}\left(Q_{1}, Q_{2}\right)$, then $f \multimap g \in \operatorname{CLin}\left(\left(P_{1} \multimap Q_{1}\right),\left(P_{2} \multimap Q_{2}\right)\right)$ is given by $(f \multimap$ $g)(h)=g h f$. The fact that $f \multimap g$ is a well defined linear function $\left(P_{1} \multimap Q_{1}\right) \rightarrow\left(P_{2} \multimap Q_{2}\right)$ results from the linearity of $f$ and $g$. The fact that it is continuous results from the fact that the lubs in $P_{i} \multimap Q_{i}$ are computed pointwise and from the continuity of $g$. The fact that $\|f \multimap g\| \leq 1$ results from the fact that the norms of $f$ and $g$ are $\leq 1$.

Bilinear maps. Given cones $P, Q$ and $R$, a function $f$ : $P \times Q \rightarrow R$ is bilinear and separately continuous if for all $x \in \mathcal{B P}$ and $y \in \mathcal{B} Q$, one has $f\left({ }_{-}, y\right) \in \mathrm{CLin}(P, R)$ and $f\left(x,{ }_{-}\right) \in \operatorname{CLin}(Q, R)$. We use $\operatorname{CLin}(P ; Q, R)$ for the set of these bilinear and separately continuous functions ${ }^{11}$.

Lemma 10. There is a natural bijection $\beta: \operatorname{CLin}(P, Q \multimap$ $R) \Rightarrow \mathrm{CLin}(P ; Q, R)$ of functors $\mathrm{CLin}{ }^{\mathrm{op}} \times \mathrm{CLin}^{\mathrm{op}} \times \mathrm{CLin} \rightarrow$ Set.

Proof. Let $g \in \operatorname{CLin}(P, Q \multimap R)$, we define $f: P \times Q \rightarrow R$ by $f(x, y)=g(x)(y)$. It is clear that $f$ is separately linear (that is the maps $f\left({ }_{-}, y\right)$ and $f\left(x,{ }_{-}\right)$are linear) because the algebraic operations of $Q \multimap R$ are defined pointwise, let us prove separate continuity. Let $(x(n))_{n \in \mathbb{N}}$ be monotone in $\mathcal{B} P$ and $y \in \mathcal{B} Q$. Then $f\left(\sup _{n \in \mathbb{N}} x(n), y\right)=g\left(\sup _{n \in \mathbb{N}} x(n)\right)(y)=$ $\sup _{n \in \mathbb{N}} f(x(n), y)$ because lubs of linear functions are computed pointwise in $Q \multimap R$. Let $x \in \mathcal{B P}$ and $(y(n))_{n \in \mathbb{N}}$ be monotone in $\mathcal{B Q}$, we have

$$
f\left(x, \sup _{n \in \mathbb{N}} y(n)\right)=g(x)\left(\sup _{n \in \mathbb{N}} y(n)\right)=\sup _{n \in \mathbb{N}} f(x, y(n))
$$

since the linear function $g(x)$ is continuous, hence $f \in \operatorname{CLin}(P ; Q, R)$, we set $\beta(g)=f$. Let now $f \in \operatorname{CLin}(P ; Q, R)$. Let $x \in \mathcal{B} P$, then we set $g(x)=f\left(x,{ }_{-}\right) \in Q \multimap R$. Linearity and continuity of $g$ follow again from the fact that all the operations of $Q \multimap R$ (including lubs) are defined pointwise. Let $g=\beta^{\prime}(f) \in \operatorname{CLin}(P, Q \multimap R)$. It is clear that $\beta$ and $\beta^{\prime}$ are natural and inverse of each other.

\footnotetext{
${ }^{11}$ Actually separate continuity is equivalent to continuity on $P \times Q$ because our notion of continuity is defined as preservation of lubs of bounded monotone families.
} 


\subsection{Probabilistic coherence spaces}

Let $I$ be a set (that we can assume to be at most countable). Given $i \in I$, we use $e_{i}$ for the element of $\left(\mathbb{R}_{\geq 0}\right)^{I}$ such that $\left(e_{i}\right)_{j}=\delta_{i, j}$.

Given $u, u^{\prime} \in\left(\mathbb{R}_{\geq 0}\right)^{I}$, we $\operatorname{set}^{12}\left\langle u, u^{\prime}\right\rangle=\sum_{i \in I} u_{i} u_{i}^{\prime} \in \mathbb{R}_{\geq 0} \cup$ $\{\infty\}$. Given $\mathcal{U} \subseteq\left(\mathbb{R}_{\geq 0}\right)^{I}$, we set $\mathcal{U}^{\perp}=\left\{u^{\prime} \in\left(\mathbb{R}_{\geq 0}\right)^{I} \mid \forall u \in\right.$ $\left.\mathcal{U}\left\langle u, u^{\prime}\right\rangle \leq 1\right\}$. A probabilistic coherence space (PCS) is a pair $X=(|X|, \mathrm{P} X)$ where $|X|$ is a set (which can be assumed at most countable) and $\mathrm{P} X \subseteq\left(\mathbb{R}_{\geq 0}\right)^{|X|}$ such that $\mathrm{P} X=\mathrm{P} X^{\perp \perp}$ and $\forall a \in|X| 0<\sup \left\{u_{a} \mid u \in \mathrm{P} X\right\}<\infty$, the purpose of this second condition being of keeping all coefficients finite. We set $\overline{\mathrm{P}} X=\left\{u \in\left(\mathbb{R}_{\geq 0}\right)^{|X|} \mid \exists \lambda>0 \lambda u \in \mathrm{P} X\right\}$. Equipped with algebraic operations defined pointwise, it is a cancellative $\mathbb{R}_{\geq 0}$-semimodule. We define a norm by setting $\|u\|=\sup \left\{\left\langle u, u^{\prime}\right\rangle \mid u^{\prime} \in \mathrm{P} X^{\perp}\right\}$ and it is easily checked that this turns $\overline{\mathrm{P}} X$ into a separated complete cone such that $\mathcal{B}(\overline{\mathrm{P}} X)=\mathrm{P} X$.

We use $N$ for the PCS defined by $|\mathrm{N}|=\mathbb{N}$ and $\mathrm{PN}=\{u \in$ $\left.\left(\mathbb{R}_{\geq 0}\right)^{\mathbb{N}} \mid \sum_{n=0}^{\infty} u_{n} \leq 1\right\}$. Given PCSs $X$ and $Y$ we define a $\operatorname{PCS} X \multimap Y$ by $|X \multimap Y|=|X| \times|Y|$ and $t \in \mathrm{P}(X \multimap Y)$ if for all $u \in \mathrm{P} X$, one has $t u \in \mathrm{PY}$ where $(t u)_{b}=\sum_{a \in|X|} t_{a, b} u_{a}$ (matrix application). The proof that this is indeed a PCS, as well as the proof of most of the next results can be found in [4]. Such matrices can be composed: given $s \in \mathrm{P}(X \multimap Y)$ and $t \in \mathrm{P}(Y \multimap Z), t s \in \mathrm{P}(X \multimap Z)$ is defined as an ordinary composition of (usually infinite-dimensional) matrices $(t s)_{a, c}=\sum_{b \in|Y|} s_{a, b} t_{b, c}$. In that way we define the category Pcoh whose objects are the PCSs and $\mathrm{P} \operatorname{coh}(X, Y)=\mathrm{P}(X \multimap$ $Y)\left(\operatorname{Id} \in \operatorname{Pcoh}(X, X)\right.$ is given by $\operatorname{Id}_{a, b}=\delta_{a, b}$ which is equal to 1 if $a=b$ and to 0 otherwise). This category is symmetric monoidal closed, and actually *autonomous, with $X \otimes Y=\left(X \multimap Y^{\perp}\right)^{\perp}$ which satisfies $|X \otimes Y|=|X| \times|Y|$ and $\mathrm{P}(X \otimes Y)=\{u \otimes v \mid u \in \mathrm{P} X \text { and } v \in \mathrm{P} Y\}^{\perp \perp}$. It is also cartesian with product of the family (at most countable) $\left(X_{i}\right)_{i \in I}$ given by $\&_{i \in I} X_{i}=X$ where $|X|=\cup_{i \in I}\{i\} \times\left|X_{i}\right|$ and $v \in \mathrm{P} X$ if $v \in\left(\mathbb{R}_{\geq 0}\right)^{|X|}$ satisfies $\forall i \in I\left(v_{i, a}\right)_{a \in\left|X_{i}\right|} \in \mathrm{P} X_{i}$ and projection $\operatorname{pr}_{i} \in \operatorname{Pcoh}\left(X, X_{i}\right)$ given by $\left(\mathrm{pr}_{i}\right)_{(j, b), a}=\delta_{j, i} \delta_{b, a}$.

PCSs as cones. There is a fully faithful functor $\bar{P}:$ Pcoh $\rightarrow$ CLin which maps a PCS $X$ to $\overline{\mathrm{P}} X$ and a matrix $t \in \operatorname{Pcoh}(X, Y)$ to the map $\overline{\mathrm{P}}(t): \overline{\mathrm{P}} X \rightarrow \overline{\mathrm{P}} Y$ defined by $\overline{\mathrm{P}}(t)(u)=t u$. We use $\mathbf{L}_{\infty}$ for the full subcategory of Pcoh whose objects are the PCSs $X$ such that $\mathrm{P} X=\left\{u \in\left(\mathbb{R}_{\geq 0}\right)^{|X|}|\forall a \in| X \mid u_{a} \leq\right.$ $1\}$. This category contains in particular the objects 1 (with $|1|=\{*\}), \mathrm{N}^{\perp}$ and is closed under \&. Notice that Pcoh (and hence $\mathbf{L}_{\infty}$ ) is essentially small since we only consider PCSs with at most countable webs so we can assume that their webs are all subsets of $\mathbb{N}$ (in the sequel we consider $\mathbf{L}_{\infty}$ as small). We use $\bar{P}_{\infty}$ for the inclusion functor $L_{\infty} \rightarrow$ CLin (it is simply the restriction of $\bar{P}$, so quite often we will drop the subscript $\infty$ ).

\footnotetext{
${ }^{12}$ This notation is compatible with our previous use of $\left\langle_{\iota_{-}}\right\rangle$in the context of cones as $u^{\prime}$ can be thought of as a linear form acting on $u$.
}

Lemma 11. Let $I$ be an at most countable set and let $\mathcal{U} \subseteq$ $\left(\mathbb{R}_{\geq 0}\right)^{I}$ be such that $\forall a \in I 0<\sup \left\{u_{a} \mid u \in \mathcal{U}\right\}<\infty$. Then $(I, \mathcal{U})$ is a PCS iff $\mathcal{U}$ is convex, downwards closed and closed under lubs of monotone sequences.

This characterization was already stated and sketchily proven in [11], a more detailed proof can be found in [5].

Lemma 12. Let $I$ be an at most countable set and let $\mathcal{U} \subseteq$ $\left(\mathbb{R}_{\geq 0}\right)^{I}$ be such that $\forall a \in I 0<\sup \left\{u_{a} \mid u \in \mathcal{U}\right\}<$ $\infty$. Let $P$ be a cone and let $h: I \rightarrow P$ be such that $\forall u \in$ $\mathcal{U} \sum_{a \in I} u_{a} h(a) \in \mathcal{B P}$. Then $\forall u \in \mathcal{U}^{\perp \perp} \sum_{a \in I} u_{a} h(a) \in \mathcal{B P}$ and $\bar{h}: u \mapsto \sum_{a \in I} u_{a} h(a)$ belongs to $\operatorname{CLin}\left(\overline{\mathrm{P}}\left(I, \mathcal{U}^{\perp \perp}\right), P\right)$.

Proof. Let $G \subseteq\left(\mathbb{R}_{\geq 0}\right)^{I}$. Let $\operatorname{cvx}(\mathcal{G})$ be the set of all the elements of $\left(\mathbb{R}_{\geq 0}\right)^{I}$ which are of shape $\sum_{j=1}^{k} \alpha_{j} u(j)$ where $u(j) \in \mathcal{G}$ and $\sum_{j=1}^{k} \alpha_{j}=1$. We use $\mathcal{G}^{+}$for the set of all $u \in$ $\left(\mathbb{R}_{\geq 0}\right)^{I}$ such that there is a monotone sequence $(u(n))_{n \in \mathbb{N}}$ of elements of $\operatorname{cvx}(\mathcal{G})$ such that $u \leq \sup _{n \in \mathbb{N}} u(n)$. Clearly $\mathcal{G} \subseteq \mathcal{G}^{+}$. For each ordinal $\beta$, we define $\mathcal{U}(\beta) \subseteq\left(\mathbb{R}_{\geq 0}\right)^{I}$ by induction as follows: $\mathcal{U}(0)=\mathcal{U}, \mathcal{U}(\beta+1)=\mathcal{U}(\beta)^{+}$and, if $\beta$ is limit and $>0$, then $\mathcal{U}(\beta)=\cup_{\gamma<\beta} \mathcal{U}(\gamma)$. This sequence is clearly monotone for $\subseteq$. Let $\beta$ be the least ordinal number such that $\mathcal{U}(\beta+1)=\mathcal{U}(\beta)$. We have $\mathcal{U}^{\perp \perp}=\mathcal{U}(\beta)$ since $\mathcal{U}(\beta)$ is the least subset of $\left(\mathbb{R}_{\geq 0}\right)^{I}$ which contains $\mathcal{U}$, is convex, downwards-closed and closed under the lubs of monotone sequences, and therefore satisfies $\mathcal{U}(\beta)^{\perp \perp}=\mathcal{U}(\beta)$ by Lemma 11.

To prove our contention, it suffices therefore to prove that, for any $G \subseteq\left(\mathbb{R}_{\geq 0}\right)^{I}$ and any $h: I \rightarrow P$ such that $\forall u \in \mathcal{G} \sum_{a \in I} u_{a} h(a) \in \mathcal{B P}$, one has $\forall u \in \mathcal{G}^{+} \sum_{a \in I} u_{a} h(a) \in$ $\mathcal{B P}$, the result will follow by ordinal induction. So assume that $\mathcal{G}$ and $h$ satisfy these hypotheses. First let $v \in \operatorname{cvx}(\mathcal{G})$, say $v=\sum_{j=1}^{k} \alpha_{j} v(j)$ where $v(j) \in \mathcal{G}$ and $\alpha_{j} \in \mathbb{R}_{\geq 0}$ such that $\sum_{j=1}^{k} \alpha_{j}=1$. Then

$$
\begin{aligned}
\sum_{a \in I} v_{a} h(a) & =\sum_{a \in I}\left(\sum_{j=1}^{k} \alpha_{j} v(j)_{a}\right) h(a) \\
& =\sum_{j=1}^{k} \alpha_{j}\left(\sum_{a \in I} v(j)_{a} h(a)\right) \in \mathcal{B P}
\end{aligned}
$$

by convexity ${ }^{13}$ of $\mathcal{B P}$.

Let now $u \in \mathcal{G}^{+}$and let $(u(n))_{n \in \mathbb{N}}$ be a monotone sequence in $\operatorname{cvx}(\mathcal{G})$ such that $u \leq \sup _{n \in \mathbb{N}} u(n)$. For each $n$ we have $\sum_{a \in I} u(n)_{a} h(a) \in \mathcal{B P}$ by what we have just proven and hence $\sup _{n \in \mathbb{N}} \sum_{a \in I} u(n)_{a} h(a) \in \mathcal{B P}$ by completeness of $P$ since the sequence $\left(\sum_{a \in I} u(n)_{a} h(a)\right)_{n \in \mathbb{N}}$ is monotone. By continuity of the algebraic operations in $P$ we have

$$
\sup _{n \in \mathbb{N}} \sum_{a \in I} u(n)_{a} h(a)=\sum_{a \in I} \sup _{n \in \mathbb{N}} u(n)_{a} h(a)
$$

\footnotetext{
${ }^{13}$ And actually also closeness because this computation uses implicitly re-
} strictions of the sum over $I$ to finite subsets of $I$. 
and since $\forall a \in I u_{a} \leq \sup _{n \in \mathbb{N}} u(n)_{a}$ we get $\sum_{a \in I} u_{a} h(a) \in$ $\mathcal{B P}$ as contended.

The fact that $\bar{h} \in \operatorname{CLin}\left(\overline{\mathrm{P}}\left(I, \mathcal{U}^{\perp \perp}\right), P\right)$ results clearly from its definition and from the fact that it maps $\mathcal{U}^{\perp \perp}$ to $\mathcal{B P}$.

Notice that it is not true that $\mathcal{U}^{\perp \perp}$ is the set of all (at most countable) convex combinations $\sum_{j \in J} \alpha_{j} u(j)$ for $u(j) \in \mathcal{U}$, simply because the set of these convex combinations is not downwards closed in general.

Example 13. To illustrate this fact, take $I=\{1,2\} \times\{1,2\}$ and $\mathcal{U}=\{u \otimes v \mid u, v \in \mathrm{P}(1 \& 1)\}$ so that $\left(I, \mathcal{U}^{\perp \perp}\right)=$ $(1 \& 1) \otimes(1 \& 1)$. In the set $\mathcal{V}$ of convex combinations of elements of $\mathcal{U}$ we have for instance $e_{1,1}=e_{1} \otimes e_{1}, e_{2,2}=e_{2} \otimes e_{2}$, $e_{1,1}+e_{1,2}+e_{2,1}+e_{2,2}=\left(e_{1}+e_{2}\right) \otimes\left(e_{1}+e_{2}\right)$, but we do not have $e_{1,2}+e_{2,1}$ (which cannot be obtained as a convex combination of $e_{1,2}$ and $\left.e_{2,1}\right)$. Notice that this latter element can be obtained as an iterated difference of convex combinations: $e_{1,2}+e_{2,1}=$ $\left(\left(e_{1}+e_{2}\right) \otimes\left(e_{1}+e_{2}\right)-e_{1} \otimes e_{1}\right)-e_{2} \otimes e_{2}$.

\subsection{Density of probabilistic coherence spaces}

We prove that the functor $\bar{P}_{\infty}$ is dense ${ }^{14}$, in the sense explained in Section 2. Let $P \in \mathrm{Obj}$ (CLin), the objects of the category $\overline{\mathrm{P}}_{\infty} / P$ are the pairs $(X, f)$ where $X \in \operatorname{Obj}\left(\mathbf{L}_{\infty}\right)$ and $f \in \mathrm{CLin}(\overline{\mathrm{P}} X, P)$. And $t \in\left(\overline{\mathrm{P}}_{\infty} / P\right)((X, f),(Y, g))$ means that $t \in \operatorname{Pcoh}(X, Y)$ and $g \overline{\mathrm{P}}(t)=f$. Then $\Delta_{P}$ is the first projection functor $\overline{\mathrm{P}} / P \rightarrow$ CLin mapping $(X, f)$ to $\overline{\mathrm{P}}(X)$ and $t$ to $\overline{\mathrm{P}}(t)$. And $\gamma^{P}: \Delta_{P} \Rightarrow P$ is the cocone $(X, f) \mapsto f$.

Given $x \in P$ where $P \in \operatorname{Obj}(\mathrm{CLin})$, we use $\widehat{x}$ for the element of the cone $\overline{\mathrm{P}} 1 \multimap P$ defined by $\widehat{x}(\lambda)=\lambda x$ (so that $\left.\|\widehat{x}\|_{\overline{\mathrm{P}} 1 \multimap P}=\|x\|\right)$.

Theorem 14. The functor $\overline{\mathrm{P}}_{\infty}: \mathrm{L}_{\infty} \rightarrow$ CLin is dense, that is, the cocone $\gamma^{P}$ is colimiting, for any object $P$ of CLin.

Proof. Let $\delta: \Delta_{P} \Rightarrow Q$ be another cocone. This means that for each $X \in \operatorname{Obj}\left(\mathbf{L}_{\infty}\right)$ and each $f \in \operatorname{CLin}\left(\overline{\mathrm{P}}_{\infty} X, P\right)$ we are given a $\delta(X, f) \in \operatorname{CLin}\left(\overline{\mathrm{P}}_{\infty} X, Q\right)$ such that for any $t \in \mathrm{L}_{\infty}(X, Y)$ we have the following implication of triangle commutations:

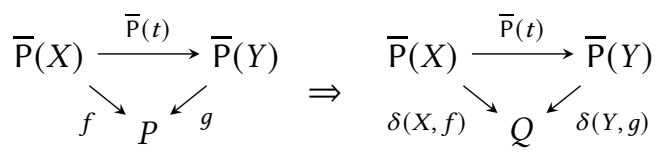

In other words for all $t \in \mathbf{L}_{\infty}(X, Y)$ and $g \in \mathbf{C L i n}(\overline{\mathrm{P}} Y, P)$

$$
\delta(X, g \overline{\mathrm{P}}(t))=\delta(Y, g) \overline{\mathrm{P}}(t) .
$$

We first build a function $k: P \rightarrow Q$ so let $x \in P$. Assume first that $\|x\| \leq 1$. Then $\widehat{x} \in \operatorname{CLin}(\overline{\mathrm{P}} 1, P)$. We set

$$
k(x)=\delta(1, \widehat{x})\left(e_{*}\right)
$$

\footnotetext{
${ }^{14}$ This is already true if we replace $\bar{P}_{\infty}$ with the full subcategory which has $\mathrm{N}^{\perp}$ as single object. Our formulation is motivated by Lemma 22 .
}

(remember that $*$ is the sole element of $|1|$ ) so that $\|k(x)\|_{Q} \leq$ 1 since $\delta(1, \widehat{x}) \in \operatorname{CLin}(\overline{\mathrm{P}} 1, Q)$. Notice that if $\lambda \in[0,1]$ we have $\lambda \operatorname{Id} \in \mathbf{L}_{\infty}(1,1)$ and hence

$$
\begin{aligned}
k(\lambda x)=\delta(1, \widehat{\lambda x})\left(e_{*}\right) & =\delta(1, \widehat{x}(\lambda \mathrm{Id}))\left(e_{*}\right) \\
& =\delta(1, \widehat{x})\left(\lambda e_{*}\right)=\lambda k(x)
\end{aligned}
$$

by (1) and linearity of $\delta(1, \widehat{x})$. Notice that we should have written $\bar{P}(\lambda$ Id) instead of $\lambda$ Id in the formulas above, we will systematically keep the $\bar{P}$ implicit $^{15}$ in this context to increase readability.

Therefore, given $x \in P$ we can set $k(x)=\lambda^{-1} k(\lambda x)$ where $\lambda \in(0,1]$ is such that $\lambda\|x\| \leq 1$; by the property we have just proven, this definition of $k(x)$ does not depend on the choice of $\lambda$. Notice that $\forall x \in P\|k(x)\| \leq\|x\|$ (since this holds when $\|x\|=1)$ and that $k(\lambda x)=\lambda k(x)$ holds for all $x \in P$ and $\lambda \in \mathbb{R}_{\geq 0}$, that is, $k$ is homogeneous.

Now we prove that the function $k$ is linear. Let $x_{1}, x_{2} \in P$, we must prove that $k\left(x_{1}+x_{2}\right)=k\left(x_{1}\right)+k\left(x_{2}\right)$. Since $k$ is homogeneous we can assume that $\left\|x_{1}\right\|+\left\|x_{2}\right\| \leq 1$. Let $a$ : $\overline{\mathrm{P}}(1 \& 1) \rightarrow P$ be defined by $a(u)=u_{1} x_{1}+u_{2} x_{2}$ (where 1,2 are the elements of $|1 \& 1|)$. This map is linear, continuous (by continuity of scalar multiplication and addition in $P$ ) and satisfies $\|a\| \leq 1$ by our assumption on the $x_{i}$ 's, hence $a \in$ $\operatorname{CLin}(\overline{\mathrm{P}}(1 \& 1), P)$.

For $i=1,2$ we have $k\left(x_{i}\right)=\delta\left(1, \widehat{x}_{i}\right)\left(e_{*}\right)=\delta\left(1, a \widehat{e}_{i}\right)\left(e_{*}\right)=$ $\delta(1 \& 1, a)\left(e_{i}\right)$ by (1) (and the fact that $\left.\widehat{x}\left(e_{*}\right)=x\right)$. Hence $k\left(x_{1}\right)+k\left(x_{2}\right)=\delta(1 \& 1, a)\left(e_{1}+e_{2}\right)$ by linearity of $\delta(1 \&$ $1, a)$. Applying again (1), as well as the definition of $a$, we get $k\left(x_{1}+x_{2}\right)=\delta\left(1, \overline{x_{1}+x_{2}}\right)\left(e_{*}\right)=\delta\left(1, a \widehat{e_{1}+e_{2}}\right)\left(e_{*}\right)=\delta(1 \&$ $1, a)\left(e_{1}+e_{2}\right)$ which proves our contention.

Next we prove that $k$ is continuous, so let $x(0) \leq x(1) \leq$ .. be a non-decreasing sequence in $\mathcal{B P}$ and let $x \in \mathcal{B P}$ be its lub. For each $n \in \mathbb{N}$ we set $y(n)=x(n)-x(n-1)$ (we set $x(-1)=0$ for convenience).

Let $u \in \overline{\mathrm{P}}\left(\mathrm{N}^{\perp}\right)$ : this means that $u \in\left(\mathbb{R}_{\geq 0}\right)^{\mathbb{N}}$ and $\sup _{n \in \mathbb{N}} u_{n}<$ $\infty$. Let $\lambda \in \mathbb{R}_{\geq 0}$ be such that $\forall n \in \mathbb{N} u_{n} \leq \lambda$. For each $N \in \mathbb{N}$ we have in $P$

$$
\sum_{n=0}^{N} u_{n} y(n) \leq \sum_{n=0}^{N} \lambda y(n)=\lambda x(N) \leq \lambda x
$$

and hence the non-decreasing sequence $\left(\sum_{n=0}^{N} u_{n} y(n)\right)_{N \in \mathbb{N}}$ has a lub in $P$ which is $\sum_{n=0}^{\infty} u_{n} y(n)$, see Section 3. So we can define a function

$$
s: \overline{\mathrm{P}}\left(\mathrm{N}^{\perp}\right) \rightarrow P, \quad u \mapsto \sum_{n=0}^{\infty} u_{n} y(n)
$$

Notice that $\forall u \in \mathrm{P}\left(\mathrm{N}^{\perp}\right)\|s(u)\| \leq\|x\| \leq 1$ since $s(u) \leq x$.

This map $s$ is linear by continuity of the algebraic operations of $P$. We prove that it is continuous so let $(u(q))_{q \in \mathbb{N}}$ be a non-decreasing sequence in $\mathrm{P}\left(\mathrm{N}^{\perp}\right)$ and let $u \in \mathrm{P}\left(\mathrm{N}^{\perp}\right)$ be its lub (that is $u_{n}=\sup _{q \in \mathbb{N}} u(q)_{n}$ for each $n \in \mathbb{N}$ ). We

\footnotetext{
${ }^{15}$ That is, consider morphisms of Pcoh as morphisms of CLin.
} 
already know that $\sup _{q \in \mathbb{N}} s(u(q)) \leq s(u)$ by linearity of $s$ (which implies monotonicity) so let us prove that $s(u) \leq$ $\sup _{q \in \mathbb{N}} s(u(q))$. This results from the fact that for any $N \in \mathbb{N}$ we have

$$
\sum_{n=0}^{N} u_{n} y(n)=\sup _{q \in \mathbb{N}} \sum_{n=0}^{N} u(q)_{n} y(n) \leq \sup _{q \in \mathbb{N}} s(u(q))
$$

where the first equation results from the continuity of the algebraic operations of $P$.

We have $\delta(1, \widehat{y(n)})\left(e_{*}\right)=\delta\left(1, s \widehat{e_{n}}\right)\left(e_{*}\right)=\delta\left(\mathrm{N}^{\perp}, s\right)\left(e_{n}\right)$ by (1) (we use also the observation that, setting $u=\lambda e_{*} \in \overline{\mathrm{P}} 1$, one has $\left(s \widehat{e_{n}}\right)(u)=s\left(\lambda e_{n}\right)=\lambda y(n)=\widehat{y(n)}(u)$, by definition of $s)$. Let $e(N)=\sum_{n=0}^{N} e_{n} \in \mathrm{P}\left(\mathrm{N}^{\perp}\right)$ so that $s(e(N))=x(N)$. We have

$$
\begin{aligned}
k(x(N)) & =k\left(\sum_{n=0}^{N} y(n)\right)=\sum_{n=0}^{N} k(y(n)) \quad \text { by linearity of } k \\
& =\sum_{n=0}^{N} \delta\left(\mathrm{N}^{\perp}, s\right)\left(e_{n}\right) \quad \text { what we have just proven } \\
& =\delta\left(\mathrm{N}^{\perp}, s\right)(e(N)) \quad \text { linearity of } \delta\left(\mathrm{N}^{\perp}, s\right)
\end{aligned}
$$

and since $\delta\left(\mathrm{N}^{\perp}, s\right)$ is continuous we have $\sup _{N \in \mathbb{N}} k(x(N))=$ $\delta\left(\mathrm{N}^{\perp}, s\right)(e)$ where $e=\sum_{n \in \mathbb{N}} e_{n}$ (that is $e_{n}=1$ for all $\left.n \in \mathbb{N}\right)$.

Next $k(x)=\delta(1, \widehat{x})\left(e_{*}\right)=\delta\left(\mathrm{N}^{\perp}, s \widehat{e}\right)\left(e_{*}\right)=\delta\left(\mathrm{N}^{\perp}, s\right)(e)$ by (1) (we use also the observation that $(s \widehat{e})(u)=s(\lambda e)=$ $\lambda x=\widehat{x}(u)$ where $u=\lambda e_{*}$, by definition of $s$ ) which proves that $k(x)=\sup _{N \in \mathbb{N}} k(x(N))$ and hence that $k$ is continuous, so $k \in \mathrm{CLin}(P, Q)$.

Now we prove that $k$ is a morphism of cocones $\Delta_{P} \Rightarrow \delta$, that is, for any $X \in \operatorname{Obj}\left(\mathbf{L}_{\infty}\right)$ and $f \in \operatorname{CLin}(\overline{\mathrm{P}} X, P)$, the following triangle commutes:

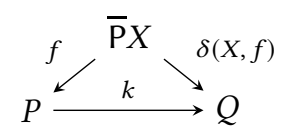

Let $u \in \overline{\mathrm{P}}(X)$, we have

$$
k(f(u))=\delta(1, \widehat{f(u)})\left(e_{*}\right)=\delta(1, f \widehat{u})\left(e_{*}\right)=\delta(X, f)(u)
$$

by (1) (we use also the observation that $(f \widehat{u})(v)=f(\lambda u)=$ $\lambda f(u)=\widehat{f(u)}(v)$, where $v=\lambda e_{*}$, by linearity of $\left.f\right)$.

We end the proof that $\gamma^{P}$ is a colimiting cocone by observing that $k$ is unique with these properties since its very definition is just a particular case of the commutation expressing that $k$ is a morphism of cocones (for $f=\widehat{x}$ with $x \in P)$.

\subsection{Completeness of the category of cones}

Theorem 15. The category CLin is complete, well-powered and admits $\mathbb{R}_{\geq 0}$ as co-generating object.

Proof. First let $\left(P_{i}\right)_{i \in I}$ be a family of cones (where $I$ is any set). We already have defined a cone $P=\prod_{i \in I} P_{i}$ as the set of all families $\vec{x}=\left(x_{i}\right)_{i \in I}$ such that $x_{i} \in P_{i}$ and $\left(\left\|x_{i}\right\|_{P_{i}}\right)_{i \in I}$ is bounded.

Equipped with the algebraic laws defined pointwise, it is a cancellative $\mathbb{R}_{\geq 0}$-semi-module. We endow it with the norm $\|\vec{x}\|=\sup _{i \in I}\left\|x_{i}\right\|_{P_{i}}$ which clearly satisfies all required axioms. The cone order of $P$ coincides with the product order which shows readily that $P$ is a complete cone.

Together with the usual projections $\mathrm{pr}_{i}: \operatorname{CLin}\left(P, P_{i}\right)$, this cone $P$ is the cartesian product of the $P_{i}$ 's as easily checked. As usual, given $f_{i} \in \operatorname{CLin}\left(Q, P_{i}\right)$ for each $i \in I$ we use $\left\langle f_{i}\right\rangle_{i \in I}$ for the morphism $f \in \operatorname{CLin}(Q, P)$ such that $f(y)=$ $\left(f_{i}(y)\right)_{i \in I}$ which is well defined by our definition of CLin which requires ${ }^{16}$ that all linear morphisms are bounded by 1. To finish we check that $P$ is separated, so let $\vec{x}, \vec{y} \in P$ be such that $\vec{x} \neq \vec{y}$. Let $i \in I$ be such that $x_{i} \neq y_{i}$. Let $x^{\prime} \in P_{i}^{\prime}$ be such that $\left\langle x_{i}, x^{\prime}\right\rangle \neq\left\langle y_{i}, x^{\prime}\right\rangle$. Then $x^{\prime} \operatorname{pr}_{i} \in P^{\prime}$ separates $\vec{x}$ from $\vec{y}$.

Let $P$ and $Q$ be cones and let $f, g \in \operatorname{CLin}(P, Q)$. Let $E=$ $\{x \in P \mid f(x)=g(x)\}$. By linearity of $f$ and $g$, this set $E$ inherits the algebraic structure of cancellative $\mathbb{R}_{\geq 0}$-semimodule from $P$. We use $e$ for the inclusion $E \subseteq P$ which is a semi-module morphism. Given $x \in E$ we set $\|x\|_{E}=\|x\|_{P}$, which clearly defines a norm on $E$. Completeness of $E$ follows from the fact that $f$ and $g$ are continuous: indeed let $(x(n))_{n \in \mathbb{N}}$ be a sequence of elements of $E$ which is nondecreasing in $E$ and hence in $P$ and satisfies $\forall n \in \mathbb{N}\|x(n)\| \leq$ 1 (for the norm of $E$, that is, for the norm of $P$ ). Let $x \in \mathcal{B} P$ be the lub of the $x(n)$ 's in $P$, by continuity of $f$ and $g$ we have $f(x)=g(x)$ and hence $x \in E$. We finish the proof by showing that $x$ is the lub of the $x(n)$ 's in $E$, so let $y \in E$ be such that $x(n) \leq_{E} y$ for all $n \in \mathbb{N}$. We have $x(n) \leq_{P} y$ and hence $x \leq_{P} y$ since $x$ is the lub of the $x(n)$ 's in $P$. By linearity of $f$ and $g$ we have $f(y-x)=f(y)-f(x)=g(y)-g(x)=g(y-x)$ and hence $y-x \in E$ which shows that $x \leq_{E} y$ as contended. The fact that $x \in \mathcal{B} E$ results obviously from the definition of the norm of $E$.

Next we prove that $E$ is separated. Let $x, y \in E$ be such that $x \neq y$. By separateness of $P$ there is an $x^{\prime} \in P^{\prime}$ such that $\left\langle x, x^{\prime}\right\rangle \neq\left\langle y, x^{\prime}\right\rangle$. Let $y^{\prime}$ be the restriction of $x^{\prime}$ to $E$, we have $y^{\prime} \in E^{\prime}$ because all operations in $E$ (including the lubs) are defined as in $P$ and of course $y^{\prime}$ separates $x$ from $y$.

Last we check that $(E, e)$ is the equalizer of $f$ and $g$ in CLin: let $h \in \operatorname{CLin}(H, P)$ be such that $f h=g h$, this means exactly that $\forall u \in H h(u) \in E$ so that we have a function $h_{0}: H \rightarrow E$ such that $h=e h_{0}$ (actually $h_{0}=h$ but it is safer to use distinct names). The linearity and continuity of $h_{0}$ results from the fact that the operations of $E$ are defined as in $P$ (including lubs). Last $h_{0}(\mathcal{B} H) \subset E \cap \mathcal{B P}=\mathcal{B} E$. Uniqueness of $h_{0}$ with these properties is obvious.

\footnotetext{
${ }^{16}$ Without this condition, the category CLin has only finite products $a$ priori.
} 
This proves that the category CLin is complete. The fact that $\mathbb{R}_{\geq 0}$ is a cogenerator results from the fact that all the objects of CLin are separated.

We are left with proving that CLin is well-powered. This results from the following simple observation.

Let $H$ be an object of CLin and $h \in \mathbf{C L i n}(H, P)$ be a mono. This implies that $h$ is an injective function. Indeed, let $u, v \in$ $H$ with $u \neq v$. Wlog. we can assume that $\|u\|,\|v\| \leq 1$. We have $\widehat{u}, \widehat{v} \in \operatorname{CLin}\left(\mathbb{R}_{\geq 0}, H\right)$ and $\widehat{u}(1) \neq \widehat{v}(1)$ hence $\widehat{u} \neq \widehat{v}$ and therefore $h \widehat{u} \neq h \widehat{v}$ from which it follows by linearity of $h \widehat{u}$ and $h \widehat{v}$ that $h(u)=h(\widehat{u}(1)) \neq h(\widehat{v}(1))=h(v)$.

Let $H_{1}=h(H)$ (so that $h$ is a bijection between $H$ and $H_{1}$ ) and equip $H_{1}$ with the addition and scalar multiplication of $P$ so that $h$ becomes an isomorphism of $\mathbb{R}_{\geq 0}$-semi-module from $H$ to $H_{1}$ (by linearity of $h$ ). We endow $H_{1}$ with the norm defined by $\|x\|_{H_{1}}=\left\|h^{-1}(x)\right\|_{H}$. The cone $H_{1}$ defined in that way is isomorphic to $H$ in our category CLin. Let $\mathcal{S}$ be the category whose objects are the objects of CLin which, as sets, are subsets of $P$ and morphisms are the monos of CLin (that is, the morphisms which are injective functions), we have shown that there is an equivalence between $\mathcal{S}$ and the category of subobjects of $P$ (by the operation $(H, h) \mapsto H_{1}$ described above), and since $\mathcal{S}$ is small (because the collection of all possible norms on a given $\mathbb{R}_{\geq 0}$-semi-module is a set, and $\mathcal{S}$ is locally small because CLin is), this shows that CLin is well-powered.

Theorem 16. Any limit-preserving functor $F:$ CLin $\rightarrow \mathrm{C}$, where the category $\mathrm{C}$ is locally small, is a right adjoint.

Proof. This is a direct application of the special adjoint functor theorem, see [14] (Chap. V, Sec. 8, Corollary).

\section{The tensor product of cones}

We use these categorical results to introduce the tensor product of cones and prove its main properties.

Lemma 17. For any given object $P$ of $\mathrm{CLin}$, the functor $P \multimap$ _ : CLin $\rightarrow$ CLin is continuous (that is, preserves all limits).

We are now in position of defining the tensor product of cones. For the time being we use a notation different from the one we used for the tensor product of PCSs.

Theorem 18. There is a unique functor $\widehat{\otimes}: \mathrm{CLin}^{2} \rightarrow \mathrm{CLin}$ such that for each $P \in \mathrm{Obj}(\mathrm{CLin})$, the functor $\_\widehat{\otimes} P$ is left adjoint to $P \multimap{ }_{-}$and that the bijection of the adjunction is natural in the three involved parameters.

Proof. By Th. 16 and Lemma 17, for each $P \in$ Obj(CLin) the functor $P \multimap{ }_{-}$has a left adjoint $\_\widehat{\otimes} P$. By the Adjunctions with a parameter theorem [14] (Chap. IV, Sec. 7), this operation extends uniquely to a functor CLin ${ }^{2} \rightarrow$ CLin in such a way that the bijection of the adjunction extends to a natural bijection $\operatorname{CLin}\left(P_{1} \widehat{\otimes} P_{2}, Q\right) \Rightarrow \operatorname{CLin}\left(P_{1}, P_{2} \multimap Q\right)$ of functors $\mathrm{CLin}^{\mathrm{op}} \times \mathrm{CLin}^{\mathrm{op}} \times \mathrm{CLin} \rightarrow$ Set.
Classification of bilinear maps. We refer to Section 3.1 for basic definitions on bilinear maps. We use cur for the natural bijection $\operatorname{CLin}(R \widehat{\otimes} P, Q) \Rightarrow \operatorname{CLin}(R, P \multimap Q)$. We set

$$
\tau_{P, Q}=\beta\left(\operatorname{cur}\left(\operatorname{Id}_{P \widehat{\otimes} Q}\right)\right) \in \operatorname{CLin}(P ; Q, P \widehat{\otimes} Q)
$$

and we use also the notation $x \widehat{\otimes} y$ for $\tau_{P, Q}(x, y) \in P \widehat{\otimes} Q$ (for $x \in P$ and $y \in Q$ ).

Theorem 19. Let $P, Q$ and $R$ be objects of CLin. For any $f \in \operatorname{CLin}(P ; Q, R)$ there is exactly one $\widehat{f} \in \operatorname{CLin}(P \widehat{\otimes} Q, R)$ such that $\widehat{f} \tau=f$.

Proof. We set $\widehat{f}=\operatorname{cur}^{-1}\left(\beta^{-1}(f)\right)$. We have

$$
\begin{aligned}
\widehat{f} \tau & =\widehat{f} \beta(\operatorname{cur}(\mathrm{Id})) \\
& =\beta((Q \multimap \widehat{f}) \operatorname{cur}(\mathrm{Id})) \quad \text { by naturality of } \beta \\
& =\beta(\operatorname{cur}(\widehat{f})) \quad \text { by naturality of cur } \\
& =f .
\end{aligned}
$$

Now we prove uniqueness so let $h \in \operatorname{CLin}(P \widehat{\otimes} Q, R)$ be such that $h \tau=f$. By the same kind of computation we have $\beta(\operatorname{cur}(h))=\beta((Q \multimap h) \operatorname{cur}(\mathrm{Id}))=h \beta(\operatorname{cur}(\mathrm{Id}))=h \tau=f$ from which it follows that $h=\widehat{f}$.

This important universal property is however not sufficient for proving that $\widehat{\otimes}$ defines a monoidal structure on CLin. One might solve this problem by showing that the natural bijection $\mathrm{CLin}(P \widehat{\otimes} Q, R) \Rightarrow \mathrm{CLin}(P, Q \multimap R)$ is actually a natural isomorphism $(P \widehat{\otimes} Q \multimap R) \Rightarrow(P \multimap(Q \multimap R))$ of functors CLin ${ }^{\mathrm{op}} \times \mathrm{CLin}^{\mathrm{op}} \times \mathrm{CLin} \rightarrow \mathrm{CLin}$. This almost works, the only non trivial point seems to be the fact that the inverse of this map has norm $\leq 1$ (we would probably need more information about the elements of $\mathcal{B}(P \widehat{\otimes} Q)$ ).

Action of $\widehat{\otimes}$ on probabilistic coherence spaces. We use another method, based on the density of PCSs in cones that we have proven; on the way we also learn that our new tensor product coincides with the old one on PCSs.

Theorem 20. There is a natural isomorphism

$$
\pi_{X, Y}: \overline{\mathrm{P}}(X \otimes Y) \Rightarrow(\overline{\mathrm{P}} X \widehat{\otimes} \overline{\mathrm{P}} Y)
$$

of functors Pcoh $^{2} \rightarrow$ CLin.

Proof. Define $\theta \in \operatorname{CLin}(\overline{\mathrm{P}} X ; \overline{\mathrm{P}} Y, \overline{\mathrm{P}}(X \otimes Y))$ by $\theta(u, v)=u \otimes v$ (it is the bilinear continuous map associated with the canonical morphism $X \multimap(Y \multimap X \otimes Y)$ in Pcoh). By Theorem 19 we have an associated $\widehat{\theta} \in \mathrm{CLin}(\overline{\mathrm{P}} X \widehat{\otimes} \overline{\mathrm{P}} Y, \overline{\mathrm{P}}(X \otimes Y))$. Now we define $\rho \in \mathrm{CLin}(\overline{\mathrm{P}}(X \otimes Y), \overline{\mathrm{P}} X \widehat{\otimes} \overline{\mathrm{P}} Y)$.

Remember that $\mathrm{P}(X \otimes Y)=\{u \otimes v \mid u \in \mathrm{P} X$ and $v \in$ $\mathrm{PY}\}^{\perp \perp}$ (warning: $u \otimes v$ is the element of $\mathrm{P}(X \otimes Y$ ) defined by $(u \otimes v)_{a, b}=u_{a} v_{b}$, not to be confused, for the time being, with $u \widehat{\otimes} v \in \overline{\mathrm{P}} X \widehat{\otimes} \overline{\mathrm{P}} Y$ ). Given $u \in \mathrm{P} X$ and $v \in \mathrm{P} Y$ we have

$$
\sum_{a \in|X|, b \in|Y|}(u \otimes v)_{a, b} \tau\left(e_{a}, e_{b}\right)=u \widehat{\otimes} v \in \mathcal{B}(\overline{\mathrm{P}} X \widehat{\otimes} \overline{\mathrm{P}} X)
$$


by bilinearity and separate continuity of $\tau$. By Lemma 12

$$
\sum_{a \in|X|, b \in|Y|} w_{a, b} \tau\left(e_{a}, e_{b}\right) \in \mathcal{B}(\overline{\mathrm{P}} X \widehat{\otimes} \overline{\mathrm{P}} X)
$$

for all $w \in \mathrm{P}(X \otimes Y)$ and the map

$$
\rho: w \mapsto \sum_{a \in|X|, b \in|Y|} w_{a, b} \tau\left(e_{a}, e_{b}\right)
$$

is linear and continuous $\overline{\mathrm{P}}(X \otimes Y) \rightarrow \overline{\mathrm{P}} X \widehat{\otimes} \overline{\mathrm{P}} Y$ and $\|\rho\| \leq 1$. For $a \in|X|$ and $b \in|Y|$, we have $\widehat{\theta}\left(\rho\left(e_{a, b}\right)\right)=\widehat{\theta}\left(e_{a} \widehat{\otimes} e_{b}\right)=$ $e_{a} \otimes e_{b}=e_{a, b}$ so that $\widehat{\theta} \rho=$ Id by linearity and continuity. Next for $u \in \mathrm{P} X$ and $v \in \mathrm{PY}$ we have $\rho \widehat{\theta} \tau(u, v)=\rho \theta(u, v)=$ $\rho(u \otimes v)=u \widehat{\otimes} v=\tau(u, v)$ and hence $\rho \widehat{\theta}=$ Id by the uniqueness part of the universal property satisfied by $\tau$. Naturality of $\widehat{\theta}$ follows from its definition.

Cocontinuity of $\widehat{\otimes}$. There is a natural transformation

$$
\sigma_{P_{1}, P_{2}, Q}^{\multimap} \in \operatorname{CLin}\left(P_{1} \multimap\left(P_{2} \multimap Q\right), P_{2} \multimap\left(P_{1} \multimap Q\right)\right)
$$

(of functors CLin ${ }^{\mathrm{op}} \times \mathrm{CLin}^{\mathrm{op}} \times \mathrm{CLin} \rightarrow$ CLin) from which we derive a natural isomorphism $\varphi_{P_{1}, P_{2}, Q}: \operatorname{CLin}\left(P_{1} \widehat{\otimes} P_{2}, Q\right) \Rightarrow$ $\operatorname{CLin}\left(P_{2} \widehat{\otimes} P_{1}, Q\right)$ by Theorem 18 and by the fact that there is a natural isomorphism $\mathrm{CLin}(P, Q) \Rightarrow \mathrm{CLin}(1, P \multimap Q))$. By Lemma 1 we get a natural isomorphism $\widetilde{\sigma}_{P_{1}, P_{2}} \in \operatorname{CLin}\left(P_{1} \widehat{\otimes}\right.$ $\left.P_{2}, P_{2} \widehat{\otimes} P_{1}\right)$.

Theorem 21. The bifunctor $\widehat{\otimes}: \mathrm{CLin}^{2} \rightarrow$ CLin is separately cocontinuous.

Proof. Being a left adjoint, the functor _ $\widehat{\otimes} P$ is cocontinuous. By the existence of the natural isomorphism $\widetilde{\sigma}$, it follows that $\widehat{\otimes}$ is cocontinuous separately in both parameters.

\subsection{Associativity isomorphisms of the tensor product}

We lift associativity of $\otimes$ on Pcoh (more precisely on the smaller category $\mathbf{L}_{\infty}$ ) to associativity of $\widehat{\otimes}$ on CLin by density.

Lemma 22. If $X$ and $Y$ are objects of $\mathbf{L}_{\infty}$ then $X \otimes Y$ is also an object of $\mathbf{L}_{\infty}$.

Proof. For a set $I$, let $1_{I} \in\left(\mathbb{R}_{\geq 0}\right)^{I}$ be defined by $\left(1_{I}\right)_{i}=1$ for all $i \in I$. If $X$ is an object of $\mathrm{L}_{\infty}$ then $1_{|X|} \in \mathrm{PX}$ and hence $1_{|X| \times|Y|}=1_{|X|} \otimes 1_{|Y|} \in \mathrm{P}(X \otimes Y)$. If $w \in \mathrm{P}(X \otimes Y), a \in|X|$ and $b \in|Y|$, we have $w_{a, b} \leq 1$ because $e_{(a, b)} \in \mathrm{P}(X \otimes Y)^{\perp}$ since $\left\langle u \otimes v, e_{(a, b)}\right\rangle \leq 1$ for all $u \in \mathrm{P} X$ and $v \in \mathrm{P} Y$.

Given $X_{i} \in \operatorname{Obj}\left(\mathbf{L}_{\infty}\right)$ for $i=1,2,3$, we define a natural isomorphism $\alpha_{X_{1}, X_{2}, X_{3}}^{0} \in \operatorname{CLin}\left(\left(\overline{\mathrm{P}} X_{1} \widehat{\otimes} \overline{\mathrm{P}} X_{2}\right) \widehat{\otimes} \overline{\mathrm{P}} X_{3}, \overline{\mathrm{P}} X_{1} \widehat{\otimes}\right.$ $\left.\left(\overline{\mathrm{P}} X_{2} \widehat{\otimes} \overline{\mathrm{P}} X_{3}\right)\right)$ as the following composition of natural isomorphisms

$$
\begin{aligned}
& \left(\overline{\mathrm{P}} X_{1} \widehat{\otimes} \overline{\mathrm{P}} X_{2}\right) \widehat{\otimes} \overline{\mathrm{P}} X_{3} \stackrel{\pi_{X_{1}, X_{2}} \widehat{\otimes} \overline{\mathrm{P}} X_{3}}{\longrightarrow} \overline{\mathrm{P}}\left(X_{1} \otimes X_{2}\right) \widehat{\otimes} \overline{\mathrm{P}} X_{3} \\
& \overline{\mathrm{P}}\left(X_{1} \otimes\left(X_{2} \otimes X_{3}\right)\right) \longleftarrow \overline{\mathrm{P}} \alpha_{X_{1}, X_{2}, X_{3}} \quad \overline{\mathrm{P}}\left(\left(X_{1} \otimes X_{2}\right) \otimes X_{3}\right) \\
& \overline{\mathrm{P}} X_{1} \widehat{\otimes} \overline{\mathrm{P}}\left(X_{2} \otimes X_{3}\right) \stackrel{\overline{\mathrm{P}} X_{1} \widehat{\otimes} \pi_{X_{2}, X_{3}}^{-1}}{\longrightarrow} \overline{\mathrm{P}} X_{1} \widehat{\otimes}\left(\overline{\mathrm{P}} X_{2} \widehat{\otimes} \overline{\mathrm{P}} X_{3}\right)
\end{aligned}
$$

Now observe that both functors $T, T^{\prime}: \mathrm{CLin}^{3} \rightarrow$ CLin defined respectively by $T\left(P_{1}, P_{2}, P_{3}\right)=\left(P_{1} \widehat{\otimes} P_{2}\right) \widehat{\otimes} P_{3}$ and $T^{\prime}\left(P_{1}, P_{2}, P_{3}\right)=P_{1} \widehat{\otimes}\left(P_{2} \widehat{\otimes} P_{3}\right)$ (and similarly on morphisms) are separately cocontinuous, because $\widehat{\otimes}$ is separately cocontinuous, see Theorem 21. We have just exhibited a natural isomorphism $\alpha^{0}: T \overline{\mathrm{P}}^{3} \Rightarrow T^{\prime} \overline{\mathrm{P}}^{3}$. Since the functor $\overline{\mathrm{P}}$ : $\mathbf{L}_{\infty} \rightarrow$ CLin is dense by Theorem 14, we can apply Theorem 6 which shows that there is exactly one natural isomorphism $\widetilde{\alpha}: T \Rightarrow T^{\prime}$ such that $\widetilde{\alpha} \overline{\mathrm{P}}^{3}=\alpha^{0}$. In other words, there are uniquely defined natural isomorphisms $\widetilde{\alpha}_{P_{1}, P_{2}, P_{3}} \in$ $\operatorname{CLin}\left(\left(P_{1} \widehat{\otimes} P_{2}\right) \widehat{\otimes} P_{3}, P_{1} \widehat{\otimes}\left(P_{2} \widehat{\otimes} P_{3}\right)\right)$ such that, for all objects

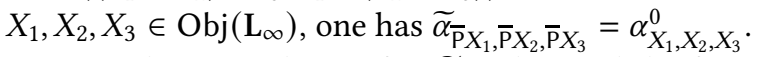

Using the naturalities of $\pi, \widetilde{\alpha}$ and $\alpha$, and the fact that $\alpha$ satisfies Mac Lane's pentagon diagram in $\mathrm{L}_{\infty}$, diagram chasing shows that $\widetilde{\alpha}$ makes the following diagram commutative for any objects $X_{i}(i=1,2,3,4)$ of $\mathbf{L}_{\infty}$

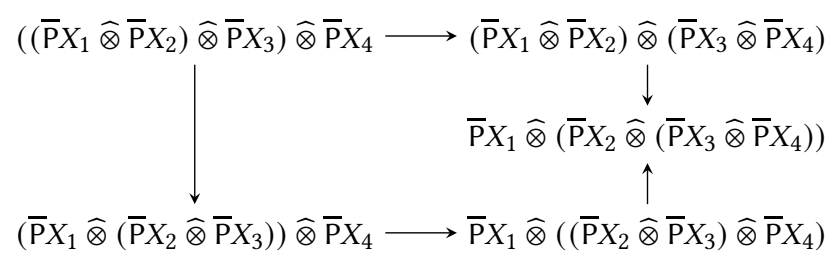

where the various morphisms are defined using $\widetilde{\alpha}$. This means that the natural isomorphisms

$$
\psi_{\vec{P}}^{1}, \psi_{\vec{P}}^{2}:\left(\left(P_{1} \widehat{\otimes} P_{2}\right) \widehat{\otimes} P_{3}\right) \widehat{\otimes} P_{4} \Rightarrow P_{1} \widehat{\otimes}\left(P_{2} \widehat{\otimes}\left(P_{3} \widehat{\otimes} P_{4}\right)\right)
$$

defined by

$$
\begin{aligned}
\psi_{\vec{P}}^{1} & =\widetilde{\alpha}_{P_{1}, P_{2}, P_{3} \widehat{\otimes} P_{4}} \widetilde{\alpha}_{P_{1} \widehat{\otimes} P_{2}, P_{3}, P_{4}} \\
\psi_{\vec{P}}^{2} & =\left(P_{1} \widehat{\otimes} \widetilde{\alpha}_{P_{2}, P_{3}, P_{4}}\right) \widetilde{\alpha}_{P_{1}, P_{1} \widehat{\otimes} P_{2}, P_{3}}\left(\widetilde{\alpha}_{P_{1}, P_{2}, P_{3}} \widehat{\otimes} P_{4}\right)
\end{aligned}
$$

satisfy $\psi_{1} \overline{\mathrm{P}}^{4}=\psi_{2} \overline{\mathrm{P}}^{4}$ and hence by the uniqueness statement of Theorem 6 we must have $\psi^{1}=\psi^{2}$, that is, $\widetilde{\alpha}$ itself satisfies Mac Lane's pentagon diagram. One deals similarly with the other coherence diagrams of symmetric monoidal categories (remember that we have defined a symmetry natural isomorphism $\widetilde{\sigma}$ in the proof of Theorem 21 , the other natural isos $\widetilde{\lambda}_{P}: 1 \widehat{\otimes} P \Rightarrow P$ and $\widetilde{\rho}_{P}: P \widehat{\otimes} 1 \Rightarrow P$ are easy to define too).

We can summarize as follows what we have proven so far.

Theorem 23. The category CLin equipped with the tensor product $\widehat{\otimes}$, the unit 1 , the natural isos $\widetilde{\lambda}, \widetilde{\rho}, \widetilde{\alpha}$ and $\widetilde{\sigma}$ is a symmetric monoidal category. It is closed, with object of morphisms 
from $P$ to $Q$ the cone $P \multimap Q$ and evaluation ev $\in \operatorname{CLin}((P \multimap Q) \widehat{\otimes}$ $P, Q)$ induced by the bilinear and continuous map $(f, x) \mapsto$ $f(x)$.

\section{The exponential}

Using again the special adjoint functor theorem, we equip CLin with a comonad !_ whose Kleisli category is (isomorphic to) our category Cstab of cones and stable functions. We start with recalling the definition of the category ${ }^{17}$.

Given $n \in \mathbb{N}$ we use $\mathcal{P}^{+}(n)$ (resp. $\left.\mathcal{P}^{-}(n)\right)$ for the set of all $I \subseteq\{1, \ldots, n\}$ such that $n-\# I$ is even (resp. odd).

Let $P$ and $Q$ be cones, in [9] is defined the notion of stable function $P \rightarrow Q$ and proven that cones equipped with these functions form a cartesian closed category. Such a function is defined only on $\mathcal{B P}$,

- is bounded (that is $\{\|f(x)\| \mid x \in \mathcal{B P}\}$ is bounded),

- totally monotone: for any $n \in \mathbb{N}$ and any $x_{1}, \ldots, x_{n} \in$ $\mathcal{B P}$ with $\sum_{i=1}^{n} x_{i} \in \mathcal{B P}$, one has $\Delta^{-} f(\vec{x}) \leq \Delta^{+} f(\vec{x})$ where $\Delta^{-} f(\vec{x})=\sum_{I \in \mathcal{P}^{-}(n)} f\left(\sum_{i \in I} x_{i}\right)$ and $\Delta^{+} f(\vec{x})=$ $\sum_{I \in \mathcal{P}^{+}(n)} f\left(\sum_{i \in I} x_{i}\right)$ (notice that the conditions for $n=$ 1,2 , namely $f(0) \leq f(x)$ and $f\left(x_{1}\right)+f\left(x_{2}\right) \leq f\left(x_{1}+\right.$ $\left.x_{2}\right)+f(0)$, imply that $f$ is monotone),

- and Scott-continuous (that is commutes with lubs of monotone sequences in $\mathcal{B P}$ ).

Equipped with algebraic operations defined pointwise and with the norm defined by $\|f\|=\sup _{x \in \mathcal{B} P}\|f(x)\|$, the set of stable functions is an object of CLin that we denote as $[P \rightarrow Q]$, separateness being proven as in the case of $P \multimap$ $Q$. We use Cstab for the category whose objects are those of CLin and morphisms are the stable functions $f$ such that $\|f\| \leq 1$.

Theorem 24 ([9]). The category Cstab is cartesian closed with cartesian product defined as in CLin, internal hom object $[P \rightarrow Q]$ and evaluation map defined as in Set.

Notice that $\operatorname{CLin}(P, Q) \subseteq \operatorname{Cstab}(P, Q)$ since linearity implies total monotonicity, this induces a "forgetful" faithful functor D : CLin $\rightarrow$ Cstab which acts as the identity on objects and morphisms. For the same reason we can consider $\left[{ }_{-} \rightarrow_{-}\right]$as a functor $\mathrm{CLin}^{\mathrm{op}} \times \mathrm{CLin} \rightarrow$ CLin defined exactly in the same way as the functor $\multimap_{-}$.

Lemma 25. With any $f \in \operatorname{CLin}(P,[Q \rightarrow R])$ we can associate an element $g$ of $\operatorname{Cstab}(Q, P \multimap R)$ defined by $g(y)(x)=$ $f(x)(y)$. This correspondence is a natural bijection of functors $\mathrm{CLin}^{\mathrm{op}} \times \mathrm{CLin}^{\mathrm{op}} \times \mathrm{CLin} \rightarrow$ Set.

Proof. Let $f \in \operatorname{CLin}(P,[Q \rightarrow R])$. Let $y \in \mathcal{B} Q$, the function $f\left({ }_{-}\right)(y): P \rightarrow R$ is linear and continuous because the algebraic operations and lubs in $[Q \rightarrow R]$ are computed

\footnotetext{
${ }^{17}$ Since we have a direct description of this $\mathrm{CCC}$, the situation might be more naturally described by the concept of linear-non-linear adjunction, see [15]; we prefer this Seely category presentation with which we are more familiar.
}

pointwise. So it makes sense to define $g$ as in the statement of the lemma, we must prove that this function is stable. First since $f$ is linear and continuous, it is bounded so let $\lambda \in \mathbb{R}_{\geq 0}$ be such that $\forall x \in \mathcal{B P}\|f(x)\|_{[Q \rightarrow R]} \leq \lambda$. This means that $\forall x \in \mathcal{B P} \forall y \in \mathcal{B} Q\|f(x)(y)\|_{Q} \leq \lambda$. Therefore $\forall y \in \mathcal{B} Q\|g(y)\|_{P \rightarrow R} \leq \lambda$. Next we prove that $g$ is totally monotone so let $y_{1}, \ldots, y_{n} \in \mathcal{B} Q$ be such that $\sum_{i=1}^{n} y_{i} \in \mathcal{B} Q$. Let $x \in \mathcal{B P}$, we have

$$
\begin{aligned}
\left(\Delta^{-} g(\vec{y})\right)(x) & =\Delta^{-}(f(x))(\vec{y}) \quad \text { app. is lin. in the function } \\
& \leq \Delta^{+}(f(x))(\vec{y}) \quad f(x) \text { is stable } \\
& =\left(\Delta^{+} g(\vec{y})\right)(x)
\end{aligned}
$$

and hence $\Delta^{-} g(\vec{y}) \leq \Delta^{+} g(\vec{y})$ since the algebraic order of $Q \multimap R$ coincides with the pointwise order. Continuity of $g$ follows similarly from that of each $f(x)$ and from the fact that lubs are computed pointwise in $Q \multimap R$.

Conversely let $g \in \operatorname{Cstab}(Q, P \multimap R)$. Let $x \in P$ and let us check that the function $f(x)=g\left({ }_{-}\right)(x)$ is stable. Let $\lambda \in \mathbb{R}_{\geq 0}$ be such that $\forall y \in \mathcal{B Q}\|g(y)\|_{P \rightarrow R} \leq \lambda$. Then we have $\forall y \in \mathcal{B} Q\|g(y)(x)\|_{R} \leq \lambda\|x\|_{P}$ and this shows that $f(x)$ maps $\mathcal{B} Q$ to a bounded subset of $R$. Let $y_{1}, \ldots, y_{n} \in \mathcal{B} Q$ be such that $\sum_{i=1}^{n} y_{i} \in \mathcal{B} Q$, for the same reasons as above we have $\Delta^{-}(f(x))(\vec{y}) \leq \Delta^{+}(f(x))(\vec{y})$ because $\Delta^{-} g(\vec{y}) \leq$ $\Delta^{+} g(\vec{y})$ by stability of $g$. Therefore $f(x)$ is totally monotone. Continuity of $f(x)$ results from that of $g$ and from the fact that lubs are computed pointwise in $P \multimap R$. So $f(x)$ is well defined and belongs to $[Q \rightarrow R]$. Now we prove that the function $f$ is linear. Let $x_{1}, \ldots, x_{k} \in P$ and $\alpha_{1}, \ldots, \alpha_{k} \in \mathbb{R}_{\geq 0}$, we have $\forall y \in \mathcal{B} Q f\left(\sum_{j=1}^{k} \alpha_{j} x_{j}\right)(y)=\sum_{j=1}^{k} \alpha_{j} f\left(x_{j}\right)(y)$ by linearity of each $g(y)$ and hence $f\left(\sum_{j=1}^{k} \alpha_{j} x_{j}\right)=\sum_{j=1}^{k} \alpha_{j} f\left(x_{j}\right)$ because algebraic operations are defined pointwise in $[Q \rightarrow$ $R]$. Continuity of $f$ holds for a similar reason.

These two operations are obviously natural and inverse of each other.

\section{Lemma 26. The functor $\mathrm{D}$ is continuous.}

So by the special adjoint functor theorem $\mathrm{D}$ has a left adjoint E : Cstab $\rightarrow$ CLin. Let (!, der, dig) be the associated comonad (in particular $\hat{!}=\mathrm{ED}: \mathrm{CLin} \rightarrow \mathrm{CLin}$ ).

Let $\chi_{P, Q}: \operatorname{CLin}(\mathrm{E} P, Q) \rightarrow \operatorname{Cstab}(P, \mathrm{D} Q)$ be the natural bijection associated with this adjunction. We have $\operatorname{prom}_{P}=$ $\chi\left(\operatorname{Id}_{\mathrm{E} P}\right) \in \operatorname{Cstab}(P, \widehat{!} P)$ since $\mathrm{D}(\mathrm{E} P)=\widehat{!} P$; for any $x \in \mathcal{B} P$ we set $x^{\uparrow}=\operatorname{prom}_{P}(x) \in \mathcal{B}(\widehat{!} P)$. This function $\operatorname{prom}_{P}$ is the universal stable function:

Lemma 27. For any $g \in \operatorname{Cstab}(P, Q)$ there is exactly one function $\widehat{g} \in \operatorname{CLin}(\widehat{!} P, Q)$ such that $g=\widehat{g} \circ$ prom $_{P}$, that is $\forall x \in \mathcal{B P} g(x)=\widehat{g}\left(x^{\widehat{\Upsilon}}\right)$. Hence if $f_{1}, f_{2} \in \mathrm{CLin}(\widehat{!} P, Q)$ satisfy $\forall x \in \mathcal{B P} f_{1} x^{\uparrow}=f_{2} x^{\uparrow}$ then $f_{1}=f_{2}$.

Proof. The first part is an immediate consequence of the adjunction, taking $\widehat{g}=\chi_{P, Q}^{-1}(g)$ since $g \in \operatorname{Cstab}(P, \mathrm{D} Q)$. 
The second part is a consequence of the first for $g=f_{1} \circ$ $\operatorname{prom}_{P}=f_{2} \circ \operatorname{prom}_{P}$.

Lemma 28. Let $f \in \operatorname{CLin}(P, Q)$. Then $\widehat{t} f \in \operatorname{CLin}(\widehat{!} P, \widehat{!} Q)$ is characterized by $\hat{!} f x^{\hat{!}}=(f x)^{\hat{!}}$. Dereliction and digging are

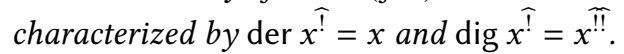

These are direct consequences of the adjunction. Given an unlabeled binary tree $B$ with $n$ leaves and $P_{1}, \ldots, P_{n} \in$ Obj(CLin), we use $B\left(P_{1}, \ldots, P_{n}\right)$ for the cone obtained by replacing each node of $B$ with the $\widehat{\otimes}$ operator and the $i$ th leaf with $\widehat{\varphi} P_{i}$. For instance if $B=\left\langle_{-},\left\langle_{-},-\right\rangle\right\rangle$then $B\left(P_{1}, P_{2}, P_{3}\right)=$ $\widehat{!} P_{1} \widehat{\otimes}\left(! P_{2} \widehat{\otimes} \widehat{!} P_{3}\right)$. We define similarly $B\left(x_{1}, \ldots, x_{n}\right)$ replacing the $i$ th leaf with $x_{i}{ }^{\uparrow}$; in the example $B\left(x_{1}, x_{2}, x_{3}\right)=x_{1}{ }^{\uparrow} \widehat{\otimes}$ $\left(x_{2}{ }^{!} \widehat{\otimes} x_{3}{ }^{\dagger}\right)$. The next statement uses these notations.

Lemma 29. Let $f_{1}, f_{2} \in \mathrm{CLin}(B(\vec{P}), Q)$ and assume that for any $x_{1} \in \mathcal{B P} P_{1}, \ldots, x_{n} \in \mathcal{B P} P_{n}$, one has $f_{1}(B(\vec{x}))=f_{2}(B(\vec{x}))$ then $f_{1}=f_{2}$.

Proof. By induction on $B$. If $B$ consists of one leaf this is just Lemma 27. Assume $B=\left\langle B_{1}, B_{2}\right\rangle$ (with $n=n_{1}+n_{2}$ and $B_{i}$ has $n_{i}$ leaves). Let $\overrightarrow{P(i)}$ be a list of cones of length $n_{i}$ (for $i=1,2$ ) and $\vec{P}$ be the concatenation of $\overrightarrow{P(1)}$ and $\overrightarrow{P(2)}$. We use similar notations for elements of these cones. We have $B(\vec{P})=$ $B_{1}(\overrightarrow{P(1)}) \widehat{\otimes} B_{2}(\overrightarrow{P(2)})$ so that cur $f_{j} \in \mathrm{CLin}\left(B_{1}(\overrightarrow{P(1)}), B_{2}(\overrightarrow{P(2)})\right.$ $Q)$ for $j=1$, 2. Let $\overrightarrow{x(1)} \in \mathcal{B} \overrightarrow{P(1)}$. For all $\overrightarrow{x(2)} \in \mathcal{B} \overrightarrow{P(2)}$ we have

$$
\begin{aligned}
\left(\operatorname{cur} f_{1}\right) & \left(B_{1}(\overrightarrow{x(1)})\right)\left(B_{2}(\overrightarrow{x(2)})\right)=f_{1}(B(\vec{x})) \\
& =f_{2}(B(\vec{x})) \quad \text { by the assumption on } f_{1} \text { and } f_{2} \\
& =\left(\operatorname{cur} f_{2}\right)\left(B_{1}(\overrightarrow{x(1)})\right)\left(B_{2}(\overrightarrow{x(2)})\right)
\end{aligned}
$$

and hence $\left(\operatorname{cur} f_{1}\right)\left(B_{1}(\overrightarrow{x(1)})\right)=\left(\operatorname{cur} f_{2}\right)\left(B_{1}(\overrightarrow{x(1)})\right)$ by inductive hypothesis applied to $B_{2}$. Next by inductive hypothesis applied to $B_{1}$ we get $\operatorname{cur} f_{1}=\operatorname{cur} f_{2}$ and hence $f_{1}=f_{2}$.

Lemma 30. There is an iso $\mathrm{m}^{0} \in \mathrm{CLin}(1, \hat{T} \mathrm{~T})$ and a natural

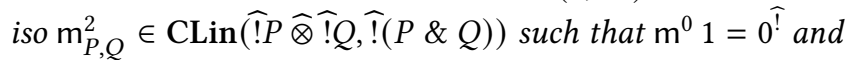
$\mathrm{m}^{2}\left(x^{\mathfrak{!}} \widehat{\otimes} y^{\widehat{!}}\right)=(x, y)^{\hat{!}}$.

Proof. We have a sequence of natural isomorphisms

$$
\begin{aligned}
\operatorname{CLin}(\widehat{!} P \widehat{\otimes} \widehat{!} Q, \widehat{!}(P \& Q)) & \\
& \Rightarrow \operatorname{CLin}(\widehat{!} P, \widehat{!} Q \multimap \widehat{!}(P \& Q)) \quad \text { by Theorem } 23 \\
& \Rightarrow \operatorname{Cstab}(P, \widehat{!} Q \multimap \widehat{!}(P \& Q)) \quad \text { since } \mathrm{E} \dashv \mathrm{D} \\
& \Rightarrow \operatorname{CLin}(\widehat{!} Q,[P \rightarrow \widehat{!}(P \& Q)]) \quad \text { by Lemma } 25 \\
& \Rightarrow \operatorname{Cstab}(Q,[P \rightarrow \widehat{!}(P \& Q)]) \\
& \Rightarrow \operatorname{Cstab}(Q \& P, \widehat{!}(P \& Q)) \\
& \Rightarrow \operatorname{Cstab}(P \& Q, \widehat{!}(P \& Q)) \quad \text { by symmetry of \& } \\
& \Rightarrow \operatorname{CLin}(\widehat{!}(P \& Q), \widehat{!}(P \& Q)) \ni \text { Id }
\end{aligned}
$$

whence a natural $\mathrm{m}_{P, Q}^{2} \in \mathrm{CLin}(\widehat{!} P \widehat{\otimes} \widehat{!} Q, \widehat{!}(P \& Q))$. This definition implies that $\mathrm{m}^{2}\left(x^{\uparrow} \widehat{\otimes} y^{\uparrow}\right)=(x, y)^{\uparrow}$. Next we define $f: \mathcal{B}(P \& Q) \rightarrow \mathcal{B}(\widehat{!} P \widehat{\otimes} \widehat{!} Q)$ by $f(x, y)=x^{!} \widehat{\otimes} y^{\dagger}$. This function is stable because prom is stable and $\widehat{\otimes}$ is bilinear and continuous. So we have $\widehat{f} \in \operatorname{CLin}(\widehat{!}(P \& Q), \widehat{!} P \widehat{\otimes} \widehat{!} Q)$ which satisfies $\widehat{f}(x, y)^{\uparrow}=x^{\grave{ }} \widehat{\otimes} y^{\uparrow}$. By Lemma 29 it follows that $\widehat{f}$ is the inverse of $\mathrm{m}^{2}$.

Since $T=\{0\}$ and $1=\mathbb{R}_{\geq 0}$ we have $g \in \operatorname{Cstab}(T, 1)$ given by $g(0)=1$ and hence $\widehat{g} \in \mathrm{CLin}(\widehat{!} \mathrm{T}, 1)$ fully characterized by $\widehat{g} 0 \widehat{!}=1$. We define $\mathrm{m}^{0} \in \mathrm{CLin}(1, \hat{!} \mathrm{T})$ by $\mathrm{m}^{0}(\lambda)=\lambda 0^{\hat{!}}$. Lemma 29 shows that $\mathrm{m}^{0} \widehat{g}=\mathrm{Id}$ and $\widehat{g} \mathrm{~m}^{0}=\mathrm{Id}$ is straightforward.

Theorem 31. Equipped with the above natural transformations (der, dig, $\mathrm{m}^{0}, \mathrm{~m}^{2}$ ), the functor $\hat{!}$ is a strong symmetric monoidal comonad from the symmetric monoidal category (CLin, \& ) to the symmetric monoidal category (CLin, $\widehat{\otimes})$ and Cstab is equivalent to the Kleisli category of this comonad.

Proof. This boils down to proving the commutation of a few diagrams (see [15]) using the above characterizations of maps by their action on tensors of elements of shape $x$ !

\section{Measurability}

We use notations introduced in Example 7. Let $\mathcal{X}$ and $\mathcal{Y}$ be measurable spaces. A substochastic kernel $\mathcal{X} \leadsto \mathcal{Y}$ is a map $K: \mathcal{X} \times \Sigma_{y} \rightarrow \mathbb{R}_{\geq 0}$ such that for each $r \in \mathcal{X}$, the map $K\left(r,{ }_{-}\right)$ is a subprobability measure on $\mathcal{Y}$ and, for each $V \in \Sigma \mathcal{y}$, the map $K\left({ }_{-}, V\right)$ is measurable. Such a kernel $K$ induces $f_{K} \in$ $\operatorname{CLin}(\underline{M}(\mathcal{X}), \underline{M}(\mathcal{Y}))$ given by $f_{K}(\mu)(V)=\int K(r, V) \mu(d r)$ from which $\bar{K}$ can be recovered since $K(r, V)=f_{K}\left(\delta_{r}\right)(V)$ (where $\delta_{r}$ is the Dirac measure at $r$ ). It is not true however that any $k \in \mathrm{CLin}(\underline{M}(\mathcal{X}), \underline{M}(\mathcal{Y}))$ allows to define a kernel $K$ by setting $K(r, V)=k\left(\delta_{r}\right)(V)$ because there is no reason for this function to be measurable in $r$.

As explained in [9] this transformation of an element of $\operatorname{CLin}(\underline{M}(\mathcal{X}), \underline{M}(\mathcal{Y}))$, and even of $\operatorname{Cstab}(\underline{M}(\mathcal{X}), \underline{M}(\mathcal{Y}))$, into a kernel $X \sim \mathcal{Y}$ is essential for interpreting the sample construct of probabilistic programming languages. This is why the objects of CLin must be equipped with an additional measurability structure and the linear and continuous morphisms must respect this structure. This set of definitions is very close in spirit to quasi-Borel spaces [19].

Let Meas be the category of measurable spaces and measurable functions and let Mref : $\mathbf{M} \rightarrow$ Meas be a functor from a cartesian reference category $\mathbf{M}$. We require Mref to preserve all finite cartesian products. The choice of this reference functor depends on the data-types of the language we want to interpret. If, as in [9], the language has the real numbers as ground type, one takes $\mathbf{M}=\mathbb{N}, \mathbf{M}(n, m)=\operatorname{Meas}\left(\mathbb{R}^{n}, \mathbb{R}^{m}\right)$, $\operatorname{Mref}(n)=\mathbb{R}^{n}$ and $\operatorname{Mref}(h)=h$ for $h \in \mathbf{M}(n, m)$. We use 0 for the terminal object of $\mathbf{M}$ (in our example it is $0 \in \mathbb{N}$ ) and, 
with this example in mind, we use + for the cartesian product in M. Hence Mref( $(0)$ is the one-point measurable space and $\operatorname{Mref}(p+q)=\operatorname{Mref}(p) \times \operatorname{Mref}(q)$. To simplify notations a little we assume that, as in our motivating example, the functor Mref acts as the identity on morphisms, that is $\mathbf{M}(p, q)=\operatorname{Meas}(\operatorname{Mref}(p), \operatorname{Mref}(q))$.

A measurable cone is a pair $P=(\underline{P}, \mathcal{M}(P))$ where $\underline{P} \in$ $\operatorname{Obj}\left(\right.$ CLin) and $\mathcal{M}(P)=\left(\mathcal{M}(P)_{p}\right)_{p \in \mathrm{M}}$ is a family of sets $\mathcal{M}(P)_{p} \subseteq\left(\underline{P}^{\prime}\right)^{\operatorname{Mref}(p)}$ whose elements satisfy ${ }^{18}:$ if $l \in \mathcal{M}(P)_{p}$ then $\forall x \in \underline{P} \lambda r l(r)(x) \in \operatorname{Meas}\left(\operatorname{Mref}(p), \mathbb{R}_{\geq 0}\right)$. Moreover this family is closed under precomposition ${ }^{19}$ by morphisms in $\mathbf{M}$ : if $l \in \mathcal{M}(P)_{p}$ then $\forall h \in \mathbf{M}(q, p) \lambda s \lambda x l(h(s))(x) \in$ $\mathcal{M}(P)_{q}$. The $l \in \mathcal{M}(P)_{p}$ are the measurability tests of arity $p$ of $P$.

A measurable path of arity $p$ of $P$ is a map $\gamma: \operatorname{Mref}(p) \rightarrow$ $\mathcal{B}(\underline{P})$ such that, for all $q \in \mathbf{M}$ and all $m \in \mathcal{M}(P)_{q}$ the function $\lambda(r, s) m(s)(\gamma(r))$ belongs to $\operatorname{Meas}\left(\operatorname{Mref}(p+q), \mathbb{R}_{\geq 0}\right)$. We use paths $s_{1}(P)_{p}$ for the set of these paths. Notice that for any $x \in \mathcal{B} \underline{P}$ one has $\lambda r x \in$ paths $_{1}(P)_{p}$ for any $p$. An $f \in \operatorname{CLin}(\underline{P}, Q)$ is measurable if $\forall \gamma \in \operatorname{paths}_{1}(P)_{p} f \circ \gamma \in$ paths $_{1}(Q)_{p}$. We use CLin ${ }_{\mathrm{m}}$ for the category of measurable cones and measurable continuous linear functions. Let $\left(P_{i}\right)_{i \in I}$ be a family of measurable cones. Given $i \in I$ and $l \in \mathcal{M}\left(P_{i}\right)_{p}$, we define $\operatorname{in}_{i}(l)$ as the element of $\left(\prod_{j \in I} P_{j}\right)^{\prime \operatorname{Mref}(p)}$ defined

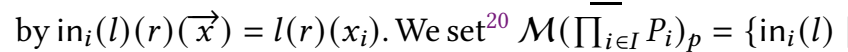
$i \in I$ and $\left.l \in \mathcal{M}\left(P_{i}\right)_{p}\right\}$ thus defining a measurable cone $\prod_{i \in I} P_{i}$ which is easily seen to be, when equipped with the ordinary projection maps, the cartesian product of the $P_{i}$ 's in $\mathrm{CLin}_{\mathrm{m}}$, so this category is cartesian ${ }^{21}$.

Let $P \multimap_{\mathrm{m}} Q$ be the cone $\mathrm{e}^{22}$ of linear and continuous functions $\overline{P \rightarrow Q}$ which are measurable in the sense that $\lambda f$ is measurable for some $\lambda>0$. It is easy to check that one turns this cone into a measurable cone $P \multimap_{\mathrm{m}} Q$ by equipping it with $\mathcal{M}\left(P \multimap \multimap_{\mathrm{m}} Q\right)_{p}=\left\{\gamma \triangleright l \mid \gamma \in \operatorname{paths}_{1}(P)_{p}\right.$ and $l \in$ $\left.\mathcal{M}(Q)_{p}\right\}$ where $\gamma \triangleright l=\lambda r \lambda f l(r)(f(\gamma(r))) \in\left(\underline{P \multimap \mathrm{m}} Q^{\prime}{ }^{\prime \operatorname{Mref}(p)}\right.$.

Given two measurable cones $P$ and $Q$, we define $P \widehat{\otimes} Q$ as the measurable cone $(\underline{P} \widehat{\otimes} Q, \mathcal{M}(P \widehat{\otimes} Q))$ where $m \in$ $(\underline{P} \widehat{\otimes} \underline{Q})^{\prime \operatorname{Mref}(k)}$ belongs to $\mathcal{M}(P \widehat{\otimes} Q)_{k}$ if for all $z \in \underline{P} \widehat{\otimes} \underline{Q}$ one has $\lambda w m(w)(z) \in \operatorname{Meas}\left(\operatorname{Mref}(k), \mathbb{R}_{\geq 0}\right)$ and for all $\gamma \bar{\epsilon}$

\footnotetext{
${ }^{18}$ It is convenient to use $\lambda_{-}$- notation borrowed to the $\lambda$-calculus to write some of the involved functions.

${ }^{19}$ This can be described in terms of presheaves of sets.

${ }^{20}$ Slightly simpler definition than in [9], but the sets of measurable paths to $\prod_{i \in I} \underline{P_{i}}$ are the same. This also explains why we have dropped the first requirement on families of sets of measurability tests.

${ }^{21}$ It is not difficult to check that it is actually small-complete by showing that it has also binary equalizers but we don't use this completeness here. ${ }^{22}$ It is easy to check that these functions equipped with the norm defined as in $P \multimap Q$, is a cone. The only point which deserves a mention is the proof of completeness which uses in a crucial way the monotone convergence theorem; as mentioned in [9] this explains why cones are complete only for bounded monotone sequences and not arbitrary directed families.
}

$\operatorname{paths}_{1}(P)_{p}$ and $\delta \in$ paths $_{1}(Q)_{q}$

$\lambda(r, s, w) m(w)(\gamma(r) \widehat{\otimes} \delta(s)) \in \operatorname{Meas}\left(\operatorname{Mref}(p+q+k), \mathbb{R}_{\geq 0}\right)$.

It is easily checked that $(\underline{P} \widehat{\otimes} \underline{Q}, \mathcal{M}(P \widehat{\otimes} Q))$ is indeed a measurable cone $P \widehat{\otimes} Q$.

Lemma 32. Let $\gamma \in$ paths $_{1}(P)_{q}$ and $\delta \in$ paths $_{1}(Q)_{q}$. Then $\lambda(r, s) \gamma(r) \widehat{\otimes} \delta(s) \in$ paths $_{1}(P \widehat{\otimes} Q)_{p+q}$, we use $\gamma \otimes \delta$ for this path.

Lemma 33. Given measurable cones $P, Q, R$, the bijection cur : $\operatorname{CLin}(\underline{P} \widehat{\otimes} \underline{Q}, \underline{R}) \rightarrow \operatorname{CLin}(\underline{P}, \underline{Q} \multimap \underline{R})$ restricts to a bijection $\operatorname{CLin}_{\mathrm{m}}(P \widehat{\otimes} Q, R) \rightarrow \operatorname{CLin}_{\mathrm{m}}\left(\bar{P}, Q \multimap \multimap_{\mathrm{m}} R\right)$.

Lemma 34. Let $f \in \operatorname{CLin}(\underline{P} \widehat{\otimes} Q, R)$. One has $f \in \operatorname{CLin}_{\mathrm{m}}(P \widehat{\otimes}$ $Q, R)$ iff for all $\gamma \in$ paths $_{1}(P)_{p}$ and $\delta \in$ paths $_{1}(Q)_{q}$, one has $f \circ(\gamma \widehat{\otimes} \delta) \in$ paths $_{1}(P \widehat{\otimes} Q)_{p+q}$.

Immediate consequence of the above. It generalizes easily, replacing $P \widehat{\otimes} Q$ with any tensorial tree like $P_{1} \widehat{\otimes}\left(P_{2} \widehat{\otimes} P_{3}\right)$. It is then routine to prove the following.

Theorem 35. The functor $\widehat{\otimes}$ restricts to a functor $\mathrm{CLin}_{\mathrm{m}}^{2} \rightarrow$ $\mathrm{CLin}_{\mathrm{m}}$ (still denoted $\widehat{\otimes}$ ). Equipped with $\widehat{\otimes}$, the category CLin is symmetric monoidal closed.

Example 36. Let $\mathcal{X}$ be a measurable space (with $\Sigma$-algebra $\left.\Sigma_{X}\right)$. Given $p \in \mathbf{M}$ and $U \in \Sigma_{X}$ we define $\varepsilon_{U} \in\left(\underline{M}(X)^{\prime}\right)^{\operatorname{Mref}(p)}$ by $\varepsilon_{U}(r)(\mu)=\mu(U)$ (for $r \in \operatorname{Mref}(p)$ ). The measurable cone $\mathrm{M}(\mathcal{X})$ is defined by $\mathrm{M}(\mathcal{X})=\underline{\mathrm{M}}(\mathcal{X})$ and $\mathcal{M}(\mathrm{M}(\mathcal{X}))_{p}=\left\{\varepsilon_{U} \mid\right.$ $\left.U \in \Sigma_{X}\right\}$. This means that paths $\mathrm{s}_{1}(\mathrm{M}(\mathcal{X}))_{p}$ is the set of all maps $\gamma: \operatorname{Mref}(p) \rightarrow \underline{M}(\mathcal{X})$ such that $\{\gamma(r)(\mathcal{X}) \mid r \in$ $\operatorname{Mref}(p)\} \subseteq \mathbb{R}_{\geq 0}$ is bounded by 1 and, for each $U \in \Sigma_{X}$, the map $\lambda r \gamma(r)(U)$ is in Meas $\left(\operatorname{Mref}(p), \mathbb{R}_{\geq 0}\right)$; in other words $\gamma: \operatorname{Mref}(p) \leadsto \mathcal{X}$. Let $K: \mathcal{X} \leadsto \mathcal{Y}$, the associated map $f_{K} \in \operatorname{CLin}(M(\mathcal{X}), M(\mathcal{Y}))$ is measurable because, given $\gamma \in$ $\mathcal{M}(\mathrm{M}(\mathcal{X}))_{p}, \overline{f_{K}} \circ \bar{\gamma}$ is nothing but the usual composition of the substochastic kernel ${ }^{23} K$ and $\gamma$. Remember that $K$ can be recovered from $f_{K}$ by $K(r)=f_{K}\left(\delta_{r}\right)$ and hence the operation $\mathcal{X} \rightarrow \mathrm{M}(\mathcal{X})$ and $K \mapsto f_{K}$ is a faithful functor $M$ from the category Kern of measurable spaces and substochastic kernels to $\mathrm{CLin}_{\mathrm{m}}$.

Conversely let $f \in \mathrm{CLin}(\mathrm{M}(\mathcal{X}), \mathrm{M}(\mathcal{Y}))$ and assume that $X=\operatorname{Mref}(p)$ for some $p \in \mathbf{M}$. Then

$$
\gamma=\lambda r \delta_{r} \in \text { paths }_{1}(\mathrm{M}(\mathcal{X}))_{p}
$$

(it is the identity kernel) and hence $K=f \circ \gamma: \mathcal{X} \leadsto$ $\mathcal{Y}$ by measurability of $f$. However the functor $M$ is not full. Take $\mathcal{Y}=1$ (the one point measurable space) so that a kernel $\mathcal{X} \leadsto \mathcal{Y}$ is simply a measurable function $\mathcal{X} \rightarrow \mathbb{R}_{\geq 0}$. Given $\mu \in \underline{M}(X)$ and $U \in \Sigma_{X}$ we define $\operatorname{disc}(\mu)(U)=\sum_{r \in U} \mu(\{r\})$ (the discrete component of $\mu$ ). Notice that, even if it is indexed by an uncountable set, this latter sum has at most countably many non-zero summands because we know that $\mu(\mathcal{X})<$ $\infty$. Then $\operatorname{disc}(\mu) \in \underline{M}(\mathcal{X})$ and it is easy to see that $\mu \mapsto$

\footnotetext{
${ }^{23}$ We are implicitly using the Giry monad.
} 
$\operatorname{disc}(\mu)$ is a linear, continuous and measurable function disc : $\underline{M}(\mathcal{X}) \rightarrow \underline{M}(\mathcal{X})$ and that disc $\leq \mathrm{Id}$. Hence $\varphi=\mathrm{Id}-\mathrm{disc}$ $\overline{i s}$ also linear, continuous and measurable $\underline{M}(\mathcal{X}) \rightarrow \underline{M}(\mathcal{X})$. Finally we define a linear, continuous and measurable function $f: \mathrm{M}(\mathcal{X}) \rightarrow \mathrm{M}\left(\mathbb{R}_{\geq 0}\right)$ by $f(\mu)=\varphi(\mu)(\mathcal{X})=\mu(\mathcal{X})-$ $\sum_{r \in \mathcal{X}} \mu(\{r\})$. In general $f \neq 0$, but by construction $f\left(\delta_{r}\right)=0$ for each $r \in \mathcal{X}$, and hence $f$ cannot be of shape $f=f_{K}$.

It seems clear that $\mathrm{M}(\mathcal{X} \times \mathcal{Y})=\mathrm{M}(\mathcal{X}) \widehat{\otimes} \mathrm{M}(\mathcal{Y})$, with $\mu \widehat{\otimes}$ $v=\mu \otimes v$ (the usual tensor product of measures), this will be checked in further work.

The exponential. We only sketch this case which is quite similar to that of $\widehat{\otimes}$. As in [9] we say that $f \in \operatorname{Cstab}(\underline{P}, Q)$ is measurable if $\forall \gamma \in$ paths $_{1}(P)_{q} f \circ \gamma \in$ paths $_{1}(Q)_{p}$. $\overline{\mathrm{W}} \mathrm{e}$ use Cstab $_{m}$ for the category of measurable cones and measurable stable functions, it is a CCC.

Let $P$ be a measurable cone. We define $\mathcal{M}(\widehat{!} P)_{q}$ as the set of all elements $l$ of $(\underline{\underline{P}})^{, \operatorname{Mref}(q)}$ such that for all $z \in \widehat{\underline{P}}$, $\lambda s l(s)(z) \in \operatorname{Meas}\left(\operatorname{Mref}(\bar{q}), \mathbb{R}_{\geq 0}\right)$ and for all $\gamma \in \operatorname{paths}_{1}(P)_{p}$, $\lambda(r, s)) l(s)\left(\gamma(r)^{\grave{!}}\right) \in \operatorname{Meas}\left(\operatorname{Mref}(p+q), \mathbb{R}_{\geq 0}\right)$. In that way, as easily checked, we have defined a measurable cone $\widehat{!} P$. If $\gamma \in$ paths $_{1}(P)_{p}$ then clearly $\gamma^{\hat{!}}=\lambda r(\gamma(r))^{\hat{!}} \in$ paths $_{1}(\widehat{!} P)_{p}$.

Theorem 37. The bijection $\mathrm{CLin}(\underline{\hat{P}}, \underline{Q}) \rightarrow \operatorname{Cstab}(\underline{P}, \underline{Q})$ restricts to a bijection $\operatorname{CLin}_{\mathrm{m}}(\widehat{!} P, Q) \rightarrow \overline{\operatorname{Cstab}}_{\mathrm{m}}(P, Q)$.

Hence, if $f \in \mathrm{CLin}(\underline{\underline{P}}, \underline{Q})$, then $f \in \mathrm{CLin}_{\mathrm{m}}(\widehat{!} P, Q)$ iff for all $\gamma \in$ paths $_{1}(P)_{p}$, it holds that $f \circ \gamma^{\hat{l}} \in$ paths $_{1}(Q)_{p}$. The required properties of $\widehat{!}_{-}$and of its associated structures follow easily.

\section{Conclusion}

We need to understand better the internal structure of $P \widehat{\otimes} Q$ and $\widehat{!} P$ (without and with measurability structure), for instance as suggested in the Introduction we conjecture that $P \widehat{\otimes} Q$ is the smallest subcone of $\operatorname{CLin}\left(P, Q ; \mathbb{R}_{\geq 0}\right)^{\prime}$ which contains all the operators $x \otimes y: f \mapsto f(x, y)$ on bilinear forms, and similarly of $\widehat{!} P$. We also conjecture that $\widehat{!} \overline{\mathrm{P}} X$ and $\overline{\mathrm{P}} ! X$ are naturally isomorphic (for PCSs $X$ ).

The framework of measurable complete positive cones seems now to be quite a general and flexible one, allowing to interpret probabilistic higher-order programming languages using continuous data types such as the real line and also general recursive data types (this feature will be presented in a forthcoming paper). With the tensor product and exponential presented here, we should be able to extend our probabilistic call-by-push-value [10] to continuous data-types. This framework also hosts naturally differential operations on programs. For instance, given a stable $f: \mathcal{B P} \rightarrow \mathbb{R}_{\geq 0}$ and elements $x, u \in \mathcal{B P}$ such that $x+u \in \mathcal{B P}$ we know thanks to [1] that the map $\varphi_{u}:[0,1] \rightarrow \mathbb{R}_{\geq 0}$, $\lambda \mapsto f(x+\lambda u)$ belongs to $\operatorname{Pcoh}(! 1,1)$ and hence has a derivative $\varphi_{u}^{\prime}(0) \in \mathbb{R}_{\geq 0}$. The map $u \mapsto \varphi_{u}^{\prime}(0)$ is linear and continuous $P_{x} \rightarrow \mathbb{R}_{\geq 0}$ (where $P_{x}$ is the "local cone" of $P$ at $x$, that is the cone of all $u \in P$ such that $x+\lambda u \in \mathcal{B P}$ for some $\lambda>0$, equipped with a suitable norm, the obvious generalization of a construction of [5] for PCSs) thus allowing to introduce a general differential calculus for stable functions on cones with expected applications in optimization as well as static analysis of programs. Of course the linear constructs on cones of this paper will be essential in these forthcoming developments.

Another interesting outcome of this work is the fact that PCSs are dense in the category CLin, a fact which might be quite useful for transferring the full abstraction results obtained so far to probabilistic programming languages handling continuous data-types. The completeness of CLin might also be quite an useful feature and an incentive for extending linear logic with dependent types; as an illustration we exhibit a natural cone which arises as an equalizer of two linear endomorphisms of a PCS.

Example 38. Let $X$ be the least solution of the equation $X=$ $1 \&(\mathrm{~N} \otimes X)$ in Pcoh in the sense explained in [4], it can be seen as a type of streams of integers. Indeed, this PCS can be described simply: $|X|$ is the set of finite sequences of integers and $u \in\left(\mathbb{R}_{\geq 0}\right)^{|X|}$ is in $\mathrm{PX}$ if $\sum_{a \in A} u_{a} \leq 1$ for all antichains $A \subseteq|X|$ (that is, set of finite sequences which are pairwise incomparable for the prefix order). Then we have a morphism $s \in \operatorname{Pcoh}(X, X)$ which is defined by $s_{a, b}=1$ if $a$ is of shape b.n $(n \in \mathbb{N}$ added at the end of the sequence $b)$ and $s_{a, b}=0$ otherwise. In other words $(s u)_{b}=\sum_{n \in \mathbb{N}} u_{b . n}$. It is not hard to see that the equalizer of $s$ and $\operatorname{Id} \in \operatorname{Pcoh}(\overline{\mathrm{P}} X, \overline{\mathrm{P}} X)$ is isomorphic to $\underline{M}(\mathcal{X})$ where $\mathcal{X}$ is the Baire space (the Polish space $\left.\mathbb{N}^{\omega}\right)$ equipped with its Borel $\Sigma$-algebra: if $s u=u$ then $u$ can be seen as the measure which maps the basic clopen set of all sequences $\in \mathbb{N}^{\omega}$ extending a to $u_{a}$. It is even possible to check that the measurability structure introduced in [1] for general PCSs seen as cones induces a measurability structure on this equalizer such that its measurable paths are exactly the substochastic kernels to $\mathcal{X}$.

This example shows that equalizers of simply definable morphisms on recursively definable types can have quite an interesting structure.

\section{Acknowledgments}

This work has been partly funded by the ANR project PPS ANR-19-CE48-0014. We thank R. Crubillé, F. Dalqvist, P.A. Melliès, M. Pagani and C. Tasson for many illuminating discussions on these topics. We are also very grateful to S. Slavnov for a communication in March 2020 where he showed us that, under some slightly stronger separatedness hypotheses on cones, the tensor product can be characterized in the way suggested in the conclusion of the present paper. This will be explained in further work. 
Last we would like to thank the reviewers of this paper for their careful reading and insightful comments and suggestions.

\section{References}

[1] Raphaëlle Crubillé. 2018. Probabilistic Stable Functions on Discrete Cones are Power Series. In Proceedings of the 33rd Annual ACM/IEEE Symposium on Logic in Computer Science, LICS 2018, Oxford, UK, July 09-12, 2018, Anuj Dawar and Erich Grädel (Eds.). ACM, 275-284.

[2] Raphaëlle Crubillé, Thomas Ehrhard, Michele Pagani, and Christine Tasson. 2017. The Free Exponential Modality of Probabilistic Coherence Spaces. In Foundations of Software Science and Computation Structures - 20th International Conference, FOSSACS 2017, Held as Part of the European Joint Conferences on Theory and Practice of Software, ETAPS 2017, Uppsala, Sweden, April 22-29, 2017, Proceedings (Lecture Notes in Computer Science, Vol. 10203), Javier Esparza and Andrzej S. Murawski (Eds.). 20-35. https://doi.org/10.1007/978-3-662-54458-7_2

[3] Fredrik Dahlqvist and Dexter Kozen. 2020. Semantics of higher-order probabilistic programs with conditioning. Proc. ACM Program. Lang. 4, POPL (2020), 57:1-57:29. https://doi.org/10.1145/3371125

[4] Vincent Danos and Thomas Ehrhard. 2011. Probabilistic coherence spaces as a model of higher-order probabilistic computation. Information and Computation 152, 1 (2011), 111-137.

[5] Thomas Ehrhard. 2019. Differentials and Distances in Probabilistic Coherence Spaces. In 4th International Conference on Formal Structures for Computation and Deduction, FSCD 2019, June 24-30, 2019, Dortmund, Germany. (LIPIcs, Vol. 131), Herman Geuvers (Ed.). Schloss Dagstuhl - Leibniz-Zentrum fuer Informatik, 17:1-17:17. https://doi.org/10.4230/LIPIcs.FSCD.2019.17

[6] Thomas Ehrhard, Michele Pagani, and Christine Tasson. 2011. The computational meaning of probabilistic coherent spaces. In Proceedings of the 26th Annual IEEE Symposium on Logic in Computer Science, LICS 2011, June 21-24, 2011, Toronto, Ontario, Canada. IEEE Computer Society, 87-96. https://doi.org/10.1109/LICS.2011.29

[7] Thomas Ehrhard, Michele Pagani, and Christine Tasson. 2014. Probabilistic coherence spaces are fully abstract for probabilistic PCF. In POPL, Suresh Jagannathan and Peter Sewell (Eds.). ACM, 309-320.

[8] Thomas Ehrhard, Michele Pagani, and Christine Tasson. 2018. Full Abstraction for Probabilistic PCF. Journal of the ACM 65, 4 (2018), 23:1-23:44.

[9] Thomas Ehrhard, Michele Pagani, and Christine Tasson. 2018. Measurable cones and stable, measurable functions: a model for probabilistic higher-order programming. Proc. ACM Program. Lang. 2, POPL (2018), 59:1-59:28. https://doi.org/10.1145/3158147

[10] Thomas Ehrhard and Christine Tasson. 2019. Probabilistic call by push value. Logical Methods in Computer Science Volume 15, Issue 1 (Jan. 2019). https://doi.org/10.23638/LMCS-15(1:3)2019

[11] Jean-Yves Girard. 2004. Between logic and quantic: a tract. In Linear Logic in Computer Science (London Mathematical Society Lecture Notes Series, Vol. 316), Thomas Ehrhard, Jean-Yves Girard, Paul Ruet, and Philip Scott (Eds.). Cambridge University Press, 346-381.

[12] Jean Goubault-Larrecq. 2015. Full abstraction for non-deterministic and probabilistic extensions of PCF I: The angelic cases. Fournal of Logical and Algebraic Methods in Programming 84, 1 (2015), 155-184. https://doi.org/10.1016/j.jlamp.2014.09.003

[13] Klaus Keimel and Gordon D. Plotkin. 2017. Mixed powerdomains for probability and nondeterminism. Logical Methods in Computer Science 13, 1 (2017).

[14] Saunders Mac Lane. 1971. Categories for the Working Mathematician. Graduate Texts in Mathematics, Vol. 5. Springer-Verlag.

[15] Paul-André Melliès. 2009. Categorical semantics of linear logic. Panoramas et Synthèses 27 (2009).
[16] Gordon Plotkin. 1977. LCF considered as a programming language. Theoretical Computer Science 5 (1977), 223-256.

[17] Peter Selinger. 2004. Towards a semantics for higher-order quantum computation. In Proceedings of the 2nd International Workshop on Quantum Programming Languages, Turku, Finland (TUCS General Publication, 33). Turku Centre for Computer Science.

[18] Serguei Slavnov. 2019. Linear logic in normed cones: probabilistic coherence spaces and beyond. Technical Report. Higher School of Economics, MIEM Faculty of Applied Mathematics and Cybernetics.

[19] Matthijs Vákár, Ohad Kammar, and Sam Staton. 2019. A domain theory for statistical probabilistic programming. Proc. ACM Program. Lang. 3, POPL (2019), 36:1-36:29.

\section{Appendix}

\subsection{Proof of Lemma 1}

Proof. We prove first naturality of $\eta$, so let $f \in \mathrm{C}\left(C, C^{\prime}\right)$, we have

$$
\begin{aligned}
F(f) \eta_{C} & =\left(\mathbf{D}(G(C), F(f)) \circ \psi_{C, F(C)}\right)\left(\operatorname{Id}_{F(C)}\right) \\
& =\left(\psi_{C, F\left(C^{\prime}\right)} \circ \mathbf{D}(F(C), F(f))\right)\left(\operatorname{Id}_{F(C)}\right) \\
& =\psi_{C, F\left(C^{\prime}\right)}(F(f)) \\
& =\left(\psi_{C, F\left(C^{\prime}\right)} \circ \mathbf{D}\left(F(f), F\left(C^{\prime}\right)\right)\right)\left(\operatorname{Id}_{F\left(C^{\prime}\right)}\right) \\
& =\left(\mathbf{D}\left(G(f), F\left(C^{\prime}\right)\right) \circ \psi_{C^{\prime}, F\left(C^{\prime}\right)}\right)\left(\operatorname{Id}_{F\left(C^{\prime}\right)}\right) \\
& =\eta_{C^{\prime}} G(f)
\end{aligned}
$$

by commutation of the diagrams

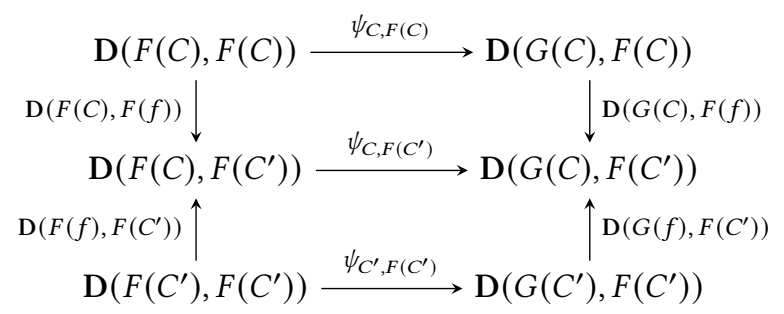

and naturality of $\theta$ is similar. Next, by naturality of $\psi$ and definition of $\theta_{C}$ we have

$$
\begin{aligned}
\theta_{C} \eta_{C} & =\theta_{C} \psi_{C, F(c)}\left(\operatorname{Id}_{F(C)}\right) \\
& \left.=\mathbf{D}\left(G(C), \theta_{C}\right) \circ \psi_{C, F(C)}\right)\left(\operatorname{Id}_{F(C)}\right) \\
& =\left(\psi_{C, G(C)} \circ \mathbf{D}\left(F(C), \theta_{C}\right)\right)\left(\operatorname{Id}_{F(C)}\right) \\
& =\psi_{C, G(C)}\left(\theta_{C}\right)=\operatorname{Id}_{G(C)}
\end{aligned}
$$

The equation $\eta_{C} \theta_{C}=\operatorname{Id}_{F(C)}$ is proven similarly.

\subsection{Proof of Lemma 2}

Proof. Let $\Delta: J \rightarrow \mathrm{C}$ be a diagram and $\gamma: \Delta \Rightarrow c$ be a colimiting cocone, we must prove that $F^{\prime} \gamma: F^{\prime} \Delta \Rightarrow F^{\prime}(c)$ is a colimiting cocone in [D, E], so let $\delta: \Delta \Rightarrow H$ be another cocone based on $\Delta$ in [D, E]. For any objects $j$ of $J$ and $d$ of D we have that $\left(\delta_{j}\right)_{d}$ (which we simply denote as $\left.\delta_{j, d}\right)$ belongs to $\mathrm{E}(F(\Delta(j), d), H(d))$ and is natural in $j$ and $d$, that is, for any $\varphi \in J\left(j, j^{\prime}\right)$ and $g \in \mathbf{D}\left(d, d^{\prime}\right)$, the following diagram 
commutes.

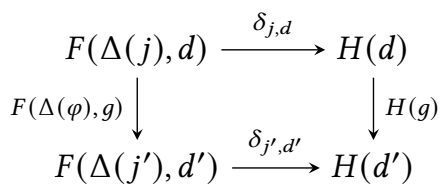

this results from the definition of $[\mathrm{D}, \mathrm{E}]$ and $F^{\prime}$.

By our assumption on $F$, for each object $d$ of $\mathbf{D}$ the $J$ cocone $F(\gamma, d): F(\Delta, d) \Rightarrow F(c, d)$ is colimiting in $\mathbf{E}$ and hence there is exactly one morphism $\theta_{d} \in \mathbf{E}(F(c, d), H(d))$ such that,

$$
\forall j \in \operatorname{Obj}(J) \quad \theta_{d} F\left(\gamma_{j}, d\right)=\delta_{j, d} .
$$

We prove that $\theta=\left(\theta_{d}\right)_{d \in \mathrm{Obj}(\mathrm{D})}$ is a natural transformation $F^{\prime}(c) \Rightarrow H$ so let $g \in \mathbf{D}\left(d, d^{\prime}\right)$, we must prove that the following diagram commutes.

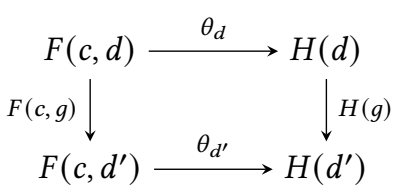

For any $j \in \operatorname{Obj}(J)$, we have

$$
\begin{aligned}
H(g) \theta_{d} F\left(\gamma_{j}, d\right) & =H(g) \delta_{j, d} \quad \text { by definition of } \theta \\
& =\delta_{j, d^{\prime}} F(\Delta(j), g) \quad \text { by naturality of } \delta \\
& =\theta_{d^{\prime}} F\left(\gamma_{j}, d^{\prime}\right) F(\Delta(j), g) \\
& =\theta_{d^{\prime}} F(c, g) F\left(\gamma_{j}, d\right)
\end{aligned}
$$

and the required commutation follows by the uniqueness part of universality from the fact that the cocone $F(\gamma, d)$ is colimiting.

This shows that $\theta \in[\mathbf{D}, \mathbf{E}]\left(F^{\prime}(c), H\right)$. It follows from (2) that for any $j \in \operatorname{Obj}(J)$, one has $\theta F^{\prime}\left(\gamma_{j}\right)=\delta$. Uniqueness follows from the fact that any $\eta \in[\mathrm{D}, \mathrm{E}]\left(F^{\prime}(c), H\right)$ such that $\eta F^{\prime}\left(\gamma_{j}\right)=\delta$ must satisfy the analogue of (2) for each given $d \in \operatorname{Obj}(D)$ and hence must be equal to $\theta$.

\subsection{Proof of Lemma 4}

Proof. Let $c \in \mathrm{C}$, for each $(x, f) \in \operatorname{Obj}(I / c)$ (so that $f \in$ $\mathrm{C}(I(x), c))$ we define $\delta_{(x, f)}:\left(F \Delta_{c}\right)(x, f)=(F I)(x) \rightarrow$ $G(c)$ by

$$
\delta_{(x, f)}=G(f) \tau_{x}
$$

(remember indeed that $\left.\tau_{x} \in \mathrm{C}((F I)(x),(G I)(x))\right)$.

Then $\delta$ is a cocone $F \Delta_{c} \Rightarrow G(c)$ because, given $t \in I / c((x, f)$, we have

$$
\begin{aligned}
\delta_{(y, g)}(F I)(t) & =G(g) \tau_{y}(F I(t)) \quad \text { by definition of } \delta \\
& =G(g)(G I)(t) \tau_{x} \quad \text { by naturality of } \tau \\
& =G(g I(t)) \tau_{x} \quad \text { by functoriality of } G \\
& =G(f) \tau_{x} \quad \text { since } t \in I / c((x, f),(y, g)) .
\end{aligned}
$$

Since the cocone $F \gamma^{c}: F \Delta_{c} \Rightarrow F(c)$ is colimiting, it follows that there is exactly one morphism $\widetilde{\tau}_{c} \in \mathrm{C}(F(c), G(c))$ such that, for each $(x, f) \in \operatorname{Obj}(I / c)$, one has $\delta_{(x, f)}=\widetilde{\tau}_{c} F\left(\gamma_{(x, f)}^{c}\right)$ that is (coming back to the definitions of $\delta$ and $\gamma^{c}$ ), the following diagram commutes

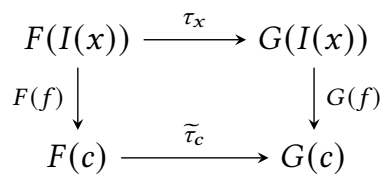

Notice that the uniqueness of this morphism implies, in the case $c=I(x)$ and $f=\mathrm{Id}$, that $\widetilde{\tau}_{x}=\tau_{x}$, so, for the first statement of the theorem, we are left with proving that $\widetilde{\tau}_{c}$ is natural in $c$. So let $h \in \mathbf{C}\left(c, c^{\prime}\right)$ and let us prove that $\tilde{\tau}_{c^{\prime}} F(h)=G(h) \widetilde{\tau}_{c}$. So let $f \in \mathbf{C}(I(x), c)$, we have

$$
\begin{aligned}
\widetilde{\tau}_{c^{\prime}} F(h) F(f) & =\widetilde{\tau}_{c^{\prime}} F(h f) \\
& =G(h f) \tau_{x} \quad \text { by definition of } \widetilde{\tau} \\
& =G(h) G(f) \tau_{x} \\
& =G(h) \tilde{\tau}_{c} F(f)
\end{aligned}
$$

and we obtain the expected commutation by Lemma 3 and the fact that $F \gamma^{c}$ is colimiting.

As to the second part of the lemma, assume that $\tau$ is a natural isomorphism whose inverse is $\sigma$, and that $G$ is also cocontinuous, we get a unique natural transformation $\widetilde{\sigma}$ : $G \Rightarrow F$ such that $\widetilde{\sigma} I=\sigma$. Now $\tilde{\tau} \widetilde{\sigma}: G \Rightarrow G$ satisfies $(\tilde{\tau} \widetilde{\sigma}) I=\tau \sigma=$ Id and hence by the uniqueness (applied to that natuarl transformation Id : $G I \Rightarrow G I$ ) we get $\widetilde{\tau} \widetilde{\sigma}=\mathrm{Id}$ and similarly $\widetilde{\sigma} \widetilde{\tau}=$ Id as contended.

\subsection{Proof of Lemma 5}

Proof. By induction on $n$, the base case $n=0$ being trivial. So for $i=1, \ldots, n$ let $I_{i}: \mathrm{C}_{i}^{0} \rightarrow \mathrm{C}_{i}$ be dense functors and let $I: \mathrm{C}^{0} \rightarrow \mathrm{C}$ be a dense functor. Let $F: \mathrm{C} \times \prod_{i=1}^{n} \mathrm{C}_{i} \rightarrow \mathrm{D}$ be a separately cocontinuous functor.

Given $c \in \operatorname{Obj}(C)$, we use $F_{c}: \prod_{i=1}^{n} \mathrm{C}_{i} \rightarrow \mathrm{D}$ for the functors obtained by fixing the first argument to $c$, notice that $F_{c}$ is separately cocontinuous.

Let $c \in \operatorname{Obj}(\mathrm{C}), \vec{c} \in \operatorname{Obj}\left(\prod_{i=1}^{n} \mathrm{C}_{i}\right), d \in \operatorname{Obj}(D)$ and let $l, l^{\prime} \in \mathrm{D}(F(c, \vec{c}), d)$ be such that for all $x \in \operatorname{Obj}\left(\mathrm{C}^{0}\right), \vec{x} \in$ $\operatorname{Obj}\left(\prod_{i=1}^{n} \mathbf{C}_{i}^{0}\right)$ and all $f \in \mathrm{C}(I(x), c)$ and $\vec{f} \in \prod_{i=1}^{n} \mathrm{C}_{i}\left(I_{i}\left(x_{i}\right), c_{i}\right)$ one has $l F(f, \vec{f})=l^{\prime} F(f, \vec{f})$.

To prove that $l=l^{\prime}$ it suffices, by inductive hypothesis ap-

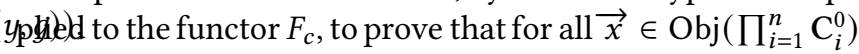
and $\vec{f} \in \prod_{i=1}^{n} \mathrm{C}_{i}\left(I_{i}\left(x_{i}\right), c_{i}\right)$ one has $l F(c, \vec{f})=l^{\prime} F(c, \vec{f})$. Let $k$ be the first of these morphisms and $k^{\prime}$ be the second one (with $\vec{x}$ and $\vec{f}$ as above). By Lemma 3 applied to the cocontinuous functor $F\left(, I_{1}\left(x_{1}\right), \ldots, I_{n}\left(x_{n}\right)\right)$ it suffices to show that for any $x \in \operatorname{Obj}\left(\mathbf{C}^{0}\right)$ and $f \in \mathbf{C}(I(x), c)$, one has $k F\left(f, I_{1}\left(x_{1}\right), \ldots, I_{n}\left(x_{n}\right)\right)=k^{\prime} F\left(f, I_{1}\left(x_{1}\right), \ldots, I_{n}\left(x_{n}\right)\right)$ which results from our assumption on $l$ and $l^{\prime}$ and functoriality of F. 


\subsection{Proof of Theorem 6}

Proof. By induction on $n$, the base case being trivial. So for $i=1, \ldots, n$ let $I_{i}: \mathrm{C}_{i}^{0} \rightarrow \mathrm{C}_{i}$ be dense functors and let $I$ : $\mathrm{C}^{0} \rightarrow \mathrm{C}$ be a dense functor. Let $F, G: \mathrm{C} \times \prod_{i=1}^{n} \mathrm{C}_{i} \rightarrow \mathrm{D}$ be functors and assume that $F$ is separately cocontinuous.

For each $x \in \operatorname{Obj}(C)^{0}$, we define a natural transformation

$$
\tau(x): F_{I(x)}\left(\prod_{i=1}^{n} I_{i}\right) \Rightarrow G_{I(x)}\left(\prod_{i=1}^{n} I_{i}\right)
$$

by setting $\tau(x)_{\vec{x}}=\tau_{x, \vec{x}}$. By inductive hypothesis, there is an unique natural transformation $\widetilde{\tau(x)}: F_{I(x)} \Rightarrow G_{I(x)}$ such that $\widetilde{\tau(x)}\left(\prod_{i=1}^{n} I_{i}\right)=\tau(x)$. So for each $x \in \operatorname{Obj}\left(\mathbf{C}^{0}\right)$, we have defined a morphism $\widetilde{\tau(x)} \in\left[F^{\prime}(I(x)), G^{\prime}(I(x))\right]$, we prove now that it is natural in $x$.

Let $t \in \mathrm{C}^{0}(x, y)$ and let $\vec{c} \in \operatorname{Obj}\left(\prod_{i=1}^{n} \mathrm{C}_{i}\right)$, we must prove that the following diagram commutes

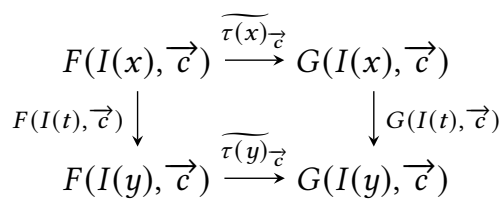

Let $\vec{x} \in \operatorname{Obj}\left(\prod_{i=1}^{n} \mathrm{C}_{i}^{0}\right)$ and $\vec{f} \in \prod_{i=1}^{n} \mathrm{C}_{i}\left(I_{i}\left(x_{i}\right), c_{i}\right)$, we have

$$
\begin{aligned}
G(I(t), \vec{c}) \widetilde{\tau(x)} \vec{c} F(I(x), \vec{f}) & \\
& =G(I(t), \vec{c}) G(I(x), \vec{f}) \widetilde{\tau(x)_{I_{1}\left(x_{1}\right), \ldots, I_{n}\left(x_{n}\right)}} \\
& =G(I(t), \vec{f}) \tau_{x, \vec{x}} \quad \text { by ind. hyp. applied to } \tau(x) \\
& =G(I(y), \vec{f}) G(I(t), \vec{x}) \tau_{x, \vec{x}} \quad \text { func. of } G \\
& =G(I(y), \vec{f}) \tau_{y, \vec{x}} F(I(t), \vec{x}) \quad \text { nat. of } \tau \\
& =\widetilde{\tau(y)_{\vec{c}}} F(I(y), f) F(I(t), \vec{x}) \quad \text { by ind. hyp. for } \tau(y) \\
& =\widetilde{\tau(y)_{\vec{c}}} F(I(t), \vec{c}) F(I(x), \vec{f})
\end{aligned}
$$

and hence by Lemma 5, $G(I(t), \vec{c}) \widetilde{\tau(x)_{\vec{c}}}=\widetilde{\tau(y)_{\vec{c}}} F(I(t), \vec{c})$ as contended.

Let $\rho: F^{\prime} I \Rightarrow G^{\prime} I$ be defined by $\rho_{x}=\widetilde{\tau(x)}$. Since $F^{\prime}$ is cocontinuous by Lemma 2 , we know by Lemma 4 that there is exactly one $\widetilde{\rho}$ such that $\widetilde{\rho} I=\rho$.

We set $\widetilde{\tau}_{c, \vec{c}}=\left(\widetilde{\rho}_{c}\right)_{\vec{c}}$, this family of morphisms $\widetilde{\tau}$ is a natural transformation $F \Rightarrow G$ such that $\widetilde{\tau}\left(I \times \prod_{i=1}^{n} I_{i}\right)=\tau$.

Uniqueness is straightforward: assume $\theta: F \Rightarrow G$ satisfies $\theta\left(I \times \prod_{i=1}^{n} I_{i}\right)=\tau$. Then $\theta_{I(x), \vec{I}(\vec{x})}=\tau(x)_{\vec{x}}$ and hence by the uniqueness of $\widetilde{\tau(x)}$ we must have $\theta_{I(x), \vec{c}}=\widetilde{\tau(x)} \vec{c}=$ $\left(\rho_{x}\right)_{\vec{c}}$. Therefore, the natural transformation $\theta^{\prime}: F^{\prime} \Rightarrow G^{\prime}$ defined by $\left(\theta_{c}^{\prime}\right)_{\vec{c}}=\theta_{c, \vec{c}}$ satisfies $\theta^{\prime} I=\rho$ from which it follows that $\theta^{\prime}=\widetilde{\rho}$, that is $\theta=\widetilde{\tau}$.

The last statement of the lemma is proven exactly as the last statement of Lemma 4.

\subsection{Proof of Lemma 9}

Proof. Let $K \subseteq I \times J$ be finite and les $K_{1} \subseteq I$ and $K_{2} \subseteq J$ be its projections, $\left\|\sum_{(i, j) \in K} x_{i, j}\right\| \leq\left\|\sum_{i \in K_{1}} \sum_{j \in K_{2}} x_{i, j}\right\|$ by monotonicity of the norm. So $\left\|\sum_{(i, j) \in K} x_{i, j}\right\| \leq\left\|\sum_{i \in K_{1}} \sum_{j \in J} x_{i, j}\right\|$ and hence the family $\left\|\sum_{(i, j) \in K} x_{i, j}\right\|_{K \in \mathcal{P}_{\text {fin }}(I \times J)}$ is bounded by our assumption that $\left(\sum_{j \in J} x_{i, j}\right)_{i \in I}$ is summable. The stated equations result from continuity of addition.

\subsection{Proof of Lemma 11}

Proof. The $\Rightarrow$ implication is easy (see [4]), we prove the converse, which uses the Hahn-Banach theorem in finite dimension. Let $v \in\left(\mathbb{R}_{\geq 0}\right)^{I}$ be such that $v \notin \mathcal{U}$. We must prove that there exists $u^{\prime} \in \mathcal{U}^{\perp}$ such that $\left\langle v, u^{\prime}\right\rangle>1$ and $\forall u \in \mathcal{U}\left\langle u, u^{\prime}\right\rangle \leq 1$. Given $J \subseteq I$ and $w \in\left(\mathbb{R}_{\geq 0}\right)^{I}$, let $\left.w\right|_{J}$ be the element of $\left(\mathbb{R}_{\geq 0}\right)^{I}$ which takes value $w_{j}$ for $j \in J$ and 0 for $j \notin J$. Then $v$ is the lub of the increasing sequence $\left\{\left.v\right|_{\left\{i_{1}, \ldots, i_{n}\right\}} \mid n \in \mathbb{N}\right\}$ (where $i_{1}, i_{2}, \ldots$ is any enumeration of I) and hence there must be some $n \in \mathbb{N}$ such that $\left.v\right|_{\left\{i_{1}, \ldots, i_{n}\right\}} \notin$ $\mathcal{U}$. Therefore it suffices to prove the result for $I$ finite, what we assume now. Let $\mathcal{G}=\left\{u \in \mathbb{R}^{I} \mid\left(\left|u_{i}\right|\right)_{i \in I} \in \mathcal{U}\right\}$ which is a convex subset of $\mathbb{R}^{I}$. Let $\lambda_{0}=\sup \left\{\lambda \in \mathbb{R}_{\geq 0} \mid \lambda v \in \mathcal{U}\right\}$. By our closeness assumption on $\mathcal{U}$, we have $\lambda_{0} v \in \mathcal{U}$ and therefore $\lambda_{0}<1$. Let $h: \mathbb{R} v \rightarrow \mathbb{R}$ be defined by $h(\lambda v)=\lambda / \lambda_{0}$ ( $\lambda_{0} \neq 0$ by our assumptions about $\mathcal{U}$ and because $I$ is finite). Let $q: \mathbb{R}^{I} \rightarrow \mathbb{R}_{\geq 0}$ be the gauge of $\mathcal{G}$, which is the semi-norm given by $q(w)=\inf \{\varepsilon>0 \mid w \in \varepsilon \mathcal{G}\}$. It is actually a norm by our assumptions on $\mathcal{U}$. Observe that $h(w) \leq q(w)$ for all $w \in \mathbb{R} v$ : this boils down to showing that $\lambda \leq \lambda_{0} q(\lambda v)=|\lambda| \lambda_{0} q(v)$ for all $\lambda \in \mathbb{R}$ which is clear since $\lambda_{0} q(v)=1$ by definition of these numbers. Hence, by the Hahn-Banach Theorem, there exists a linear $l: \mathbb{R}^{I} \rightarrow \mathbb{R}$ such that $|l| \leq q$ and which coincides with $h$ on $\mathbb{R} v$. Let $v^{\prime} \in \mathbb{R}^{I}$ be such that $\left\langle w, v^{\prime}\right\rangle=l(w)$ for all $w \in \mathbb{R}^{I}$ (using again the finiteness of $I)$. Let $u^{\prime} \in\left(\mathbb{R}_{\geq 0}\right)^{I}$ be defined by $u_{i}^{\prime}=\left|v_{i}^{\prime}\right|$. It is clear that $\left\langle v, u^{\prime}\right\rangle>1$ : since $v \in\left(\mathbb{R}_{\geq 0}\right)^{I}$ we have $\left\langle v, u^{\prime}\right\rangle \geq\left\langle v, v^{\prime}\right\rangle=l(v)=h(v)=1 / \lambda_{0}>1$. Let $N=\left\{i \in I \mid v_{i}^{\prime}<0\right\}$. Given $w \in \mathcal{U}$, let $\bar{w} \in \mathbb{R}^{I}$ be given by $\bar{w}_{i}=-w_{i}$ if $i \in N$ and $\bar{w}_{i}=w_{i}$ otherwise. Then $\left\langle w, v^{\prime}\right\rangle=\left\langle\bar{w}, u^{\prime}\right\rangle=l(\bar{w}) \leq 1$ since $\bar{w} \in \mathcal{G}$ (by definition of $\mathcal{G}$ and because $w \in \mathcal{U})$. It follows that $u^{\prime} \in \mathcal{U}^{\perp}$.

\subsection{Proof of Lemma 12}

Proof. Let $\mathcal{G} \subseteq\left(\mathbb{R}_{\geq 0}\right)^{I}$. Let $\operatorname{cvx}(\mathcal{G})$ be the set of all the elements of $\left(\mathbb{R}_{\geq 0}\right)^{I}$ which are of shape $\sum_{j=1}^{k} \alpha_{j} u(j)$ where $u(j) \in \mathcal{G}$ and $\sum_{j=1}^{k} \alpha_{j}=1$. We use $\mathcal{G}^{+}$for the set of all $u \in$ $\left(\mathbb{R}_{\geq 0}\right)^{I}$ such that there is a monotone sequence $(u(n))_{n \in \mathbb{N}}$ of elements of $\operatorname{cvx}(\mathcal{G})$ such that $u \leq \sup _{n \in \mathbb{N}} u(n)$. Clearly $\mathcal{G} \subseteq \mathcal{G}^{+}$. For each ordinal $\beta$, we define $\mathcal{U}(\beta) \subseteq\left(\mathbb{R}_{\geq 0}\right)^{I}$ by induction as follows: $\mathcal{U}(0)=\mathcal{U}, \mathcal{U}(\beta+1)=\mathcal{U}(\beta)^{+}$and, if $\beta$ is limit and $>0$, then $\mathcal{U}(\beta)=\cup_{\gamma<\beta} \mathcal{U}(\gamma)$. This sequence is clearly monotone for $\subseteq$. Let $\beta$ be the least ordinal number such that $\mathcal{U}(\beta+1)=\mathcal{U}(\beta)$. We have $\mathcal{U}^{\perp \perp}=\mathcal{U}(\beta)$ since 
$\mathcal{U}(\beta)$ is the least subset of $\left(\mathbb{R}_{\geq 0}\right)^{I}$ which contains $\mathcal{U}$, is convex, downwards-closed and closed under the lubs of monotone sequences, and therefore satisfies $\mathcal{U}(\beta)^{\perp \perp}=\mathcal{U}(\beta)$ by Lemma 11.

To prove our contention, il suffices therefore to prove that, for any $\mathcal{G} \subseteq\left(\mathbb{R}_{\geq 0}\right)^{I}$ and any $h: I \rightarrow P$ such that $\forall u \in$ $\mathcal{G} \sum_{a \in I} u_{a} h(a) \in \mathcal{B P}$, one has $\forall u \in \mathcal{G}^{+} \sum_{a \in I} u_{a} h(a) \in \mathcal{B P}$, the result will follow by ordinal induction. So assume that $\mathcal{G}$ and $h$ satisfy these hypotheses. First let $v \in \operatorname{cvx}(\mathcal{G})$, say $v=\sum_{j=1}^{k} \alpha_{j} v(j)$ where $v(j) \in \mathcal{G}$ and $\alpha_{j} \in \mathbb{R}_{\geq 0}$ such that $\sum_{j=1}^{k} \alpha_{j}=1$. Then

$$
\begin{aligned}
\sum_{a \in I} v_{a} h(a) & =\sum_{a \in I}\left(\sum_{j=1}^{k} \alpha_{j} v(j)_{a}\right) h(a) \\
& =\sum_{j=1}^{k} \alpha_{j}\left(\sum_{a \in I} v(j)_{a} h(a)\right) \in \mathcal{B P}
\end{aligned}
$$

by convexity ${ }^{24}$ of $\mathcal{B P}$.

Let now $u \in \mathcal{G}^{+}$and let $(u(n))_{n \in \mathbb{N}}$ be a monotone sequence in $\operatorname{cvx}(\mathcal{G})$ such that $u \leq \sup _{n \in \mathbb{N}} u(n)$. For each $n$ we have $\sum_{a \in I} u(n)_{a} h(a) \in \mathcal{B P}$ by what we have just proven and hence $\sup _{n \in \mathbb{N}} \sum_{a \in I} u(n)_{a} h(a) \in \mathcal{B P}$ by completeness of $P$ since the sequence $\left(\sum_{a \in I} u(n)_{a} h(a)\right)_{n \in \mathbb{N}}$ is monotone. By continuity of the algebraic operations in $P$ we have $\sup _{n \in \mathbb{N}} \sum_{a \in I} u(n)_{a} h(a)=\sum_{a \in I} \sup _{n \in \mathbb{N}} u(n)_{a} h(a)$ and since $\forall a \in I u_{a} \leq \sup _{n \in \mathbb{N}} u(n)_{a}$ we get $\sum_{a \in I} u_{a} h(a) \in \mathcal{B P}$ as contended.

The fact that $\bar{h} \in \mathrm{C} \operatorname{Lin}\left(\overline{\mathrm{P}}\left(I, \mathcal{U}^{\perp \perp}\right), P\right)$ results clearly from its definition and from the fact that it maps $\mathcal{U}^{\perp \perp}$ to $\mathcal{B P}$.

\subsection{Proof of Lemma 17}

Proof. It suffices to check that it preserves all small products and binary equalizers. Let first $\vec{Q}=\left(Q_{i}\right)_{i \in I}$ be a family of objects of CLin. Any element of $P \multimap \Pi \vec{Q}$ is of shape $\left\langle f_{i}\right\rangle_{i \in I}$ with $f_{i} \in P \multimap Q_{i}$ for each $i$ and this defines a map $\theta_{\vec{Q}}:\left(P \multimap \prod \vec{Q}\right) \rightarrow \prod_{i \in I}\left(P \multimap Q_{i}\right)$ which is a bijection. This map is linear and continuous because all operations are calculated pointwise (wrt. the argument of functions) and componentwise (in the product indexed by $I$ ). The fact that $\left\|\theta_{\vec{Q}}\right\|=1$ results from the fact that all the norms involved are computed as lubs in $\mathbb{R}_{\geq 0}$. To check that $\theta_{\vec{Q}}$ is an iso it suffices to check that $\theta_{\vec{Q}}^{-1}$ is continuous. Let us check this point: let $(f(n))_{n \in \mathbb{N}}$ be a non-decreasing sequence in $\mathcal{B}\left(\prod_{i \in I}(P \multimap\right.$ $\left.Q_{i}\right)$ ) so that $f(n)=\left(f(n)_{i}\right)_{i \in I}$, where $f(n)_{i} \in P \multimap Q_{i}$ and for each $i \in I$ the sequence of functions $\left(f(n)_{i}\right)_{n \in \mathbb{N}}$ is nondecreasing, and for each $x \in \mathcal{B} P$, one has $\forall n \in \mathbb{N}\left\|f(n)_{i}(x)\right\| \leq$ 1. Then $f=\sup _{n} f(n) \in \mathcal{B}\left(\prod_{i \in I}\left(P \multimap Q_{i}\right)\right)$ is characterized by $f(x)_{i}=\sup _{n \in \mathbb{N}} f(n)_{i}(x)$. On the other hand, $g=$

\footnotetext{
$\overline{{ }^{24} \text { And actually }}$ also closeness because this computation uses implicitely restrictions of the sum over $I$ to finite subsets of $I$.
}

$\theta_{\vec{Q}}^{-1}(f) \in P \multimap \prod \vec{Q}$ is given by $g(x)=\left(f(x)_{i}\right)_{i \in I}$ so that $g(x)$ is the lub in $\prod \vec{Q}$ of the sequence $(f(n)(x))_{n \in \mathbb{N}}$ and since lubs of sequences of functions are computed pointwise, this proves our contention. So $\theta_{\vec{Q}}$ is an iso in CLin and its naturality is obvious.

Next consider $f_{1}, f_{2} \in \operatorname{CLin}(Q, R)$ and let $(E, e)$ be the corresponding equalizer ( $E$ is the cone of elements $x$ of $Q$ such that $f_{1}(x)=f_{2}(x)$ and $e: E \rightarrow Q$ is the inclusion). Then $P \multimap f_{i} \in \operatorname{CLin}(P \multimap Q, P \multimap R$ ) (for $i=1,2)$ maps $h$ to $f_{i} h$. The equalizer of these two maps is the cone of all $h \in P \multimap Q$ such that $f_{1} h=f_{2} h$, that is $\forall x \in P f_{1}(h(x))=$ $f_{2}(h(x))$, equivalently $h \in P \multimap E$. And the inclusion map $(P \multimap E) \rightarrow(P \multimap Q)$ is equal to $P \multimap e$. Hence the equalizer of $P \multimap f_{1}$ and $P \multimap f_{2}$ is $(P \multimap E, P \multimap e)$ which proves that the functor $P \multimap \ldots$ preserves equalizers, and hence preserves all small limits.

\subsection{Proof of Lemma 26}

Proof. It suffices to prove that D preserves small products and binary equalizers. The first statement results from the fact that Cstab is cartesian with products defined as in CLin. Let us prove the second one so let $f_{1}, f_{2} \in \operatorname{CLin}(P, Q)$ and $(E, e)$ be the corresponding equalizer in CLin (that is $E=$ $\left\{x \in P \mid f_{1}(x)=f_{2}(x)\right\}$ and $e: E \rightarrow P$ is the obvious inclusion, see the proof of Theorem 15$)$. We prove that $(E, e)$ is the equalizer of $f_{1}$ and $f_{2}$ in Cstab so let $g \in \operatorname{Cstab}(R, P)$ be such that $f_{1} \circ g=f_{2} \circ g$, that is $\forall z \in \mathcal{B} R g(z) \in E$. Let $h: \mathcal{B R} \rightarrow E$ be defined by $h(z)=g(z)$, then $h$ is stable because $g$ is and $E$ inherits its structure from $P$ (which also entails that $h(\mathcal{B} R) \subseteq \mathcal{B} E$ since $g(\mathcal{B} R) \subseteq \mathcal{B P})$. And $h$ is the unique element of $\operatorname{Cstab}(R, E)$ such that $g=e \circ h$ which proves our contention.

\subsection{Proof of Lemma 33}

Proof. Let $f \in \operatorname{CLin}_{\mathrm{m}}(P \widehat{\otimes} Q, R)$ and $g=\operatorname{cur}(f)$, we prove that $g \in \operatorname{CLin}_{\mathrm{m}}\left(P, Q \multimap_{\mathrm{m}} R\right)$. Let first $x \in \underline{P}$, we prove that $g(x) \in Q \multimap_{\mathrm{m}} R$ so let $\delta \in \mathcal{M}(Q)_{p}$, we prove that $g(x) \circ \delta \in$

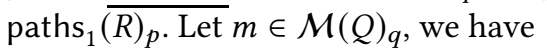

$$
\lambda(r, s) m(s)(g(x)(\delta(r)))=\lambda(r, s) m(s)(f(x \widehat{\otimes} \delta(r))) .
$$

Let $\gamma=\lambda w x \in \mathcal{M}(P)_{0}$ we have $f \circ(\gamma \widehat{\otimes} \delta) \in$ paths $_{1}(R)_{p}$ by Lemma 32 and by our assumption about $f$ and hence $\lambda(r, s) m(s)(g(x)(\delta(r))) \in \operatorname{Meas}\left(\operatorname{Mref}(p+q), \mathbb{R}_{\geq 0}\right)$ so that $g(x) \in Q \rightarrow_{\mathrm{m}} R$. We prove that $g \in \operatorname{CLin}_{\mathrm{m}}\left(P, Q \rightarrow_{\mathrm{m}}\right.$ $R)$ so let $\gamma \in$ paths $_{1}(P)_{p}$ and let us show that $g \circ \gamma \in$ paths $_{1}\left(Q \multimap_{\mathrm{m}} R\right)_{p}$; applying the definition of $Q \multimap_{\mathrm{m}} R$, let $\delta \in$ paths $_{1}(Q)_{q}$ and $l \in \mathcal{M}(R)_{q}$, we have

$$
\begin{aligned}
\lambda(r, s)(\delta \triangleright l)(s)(g(\gamma(r)) & =\lambda(r, s) l(s)(g(\gamma(r))(\delta(s))) \\
& =\lambda(r, s) l(s)(f(\gamma(r) \widehat{\otimes} \delta(s)))
\end{aligned}
$$


and we know by our assumtion on $f$ and by Lemma 32 that $f \circ(\gamma \widehat{\otimes} \delta) \in$ paths $\left._{1}(R)_{p+q}\right)$ and hence

$\lambda\left(r, s, s^{\prime}\right) l\left(s^{\prime}\right)(f(\gamma(r) \widehat{\otimes} \delta(s))) \in \operatorname{Meas}\left(\operatorname{Mref}(p+q+q), \mathbb{R}_{\geq 0}\right)$

from which $\lambda(r, s)(\delta \triangleright l)(s)\left(g(\gamma(r)) \in \operatorname{Meas}\left(\operatorname{Mref}(p+q), \mathbb{R}_{\geq 0}\right)\right.$ follows since $\mathbf{M}$ is cartesian and measurability tests are closed under precomposition by morphisms of $\mathbf{M}$.

Conversely, let $g \in \operatorname{CLin}_{\mathrm{m}}\left(P, Q \multimap_{\mathrm{m}} R\right)$ and let $f=$ $\operatorname{cur}^{-1}(g) \in \mathrm{CLin}(\underline{P} \widehat{\otimes} Q, \underline{R})$ so that $f$ is uniquely characterized by the fact that $f(x \widehat{\otimes} y)=g(x)(y)$ for all $x \in \underline{P}$ and $y \in \underline{Q}$. We must prove that $f \in \operatorname{CLin}_{\mathrm{m}}(P \widehat{\otimes} Q, R)$ so let $\theta \in$ paths $_{1}(P \widehat{\otimes} Q)_{p_{0}}$, we must show that $f \circ \theta \in$ paths $_{1}(R)_{p_{0}}$. Let $l \in \mathcal{M}(R)_{k}$ and let us prove that $\lambda\left(r_{0}, w\right) l(w)\left(f\left(\theta\left(r_{0}\right)\right) \in\right.$ $\operatorname{Meas}\left(\operatorname{Mref}\left(p_{0}+k\right), \mathbb{R}_{\geq 0}\right)$. For each $w \in \operatorname{Mref}(k)$, we have $\lambda z l(w)(f(z)) \in(\underline{P} \widehat{\otimes} Q)^{\prime}$ because $f$ is linear and continuous and $l(w) \in R^{\prime}$. So let $m \in(\underline{P} \widehat{\otimes} Q)^{\prime \operatorname{Mref}(k)}$ be defined as $m=\lambda w \lambda z l(w)(f(z))$, we claim that $m \in \mathcal{M}(P \widehat{\otimes}$ $Q)_{k}$. The first condition (namely for all $z \in \underline{P} \widehat{\otimes} Q$, one has $\left.\lambda w m(w)(z) \in \operatorname{Meas}\left(\operatorname{Mref}(k), \mathbb{R}_{\geq 0}\right)\right)$ being obviously satisfied, we check the second one so let $\gamma \in$ paths $_{1}(P)_{p}$ and $\delta \in$ paths $_{1}(Q)_{q}$. We have

$$
\begin{aligned}
\lambda(r, s, w) m(w)(\gamma(r) \widehat{\otimes} \delta(s)) & =\lambda(r, s, w) l(w)(f(\gamma(r) \widehat{\otimes} \delta(s))) \\
& =\lambda(r, s, w) l(w)(g(\gamma(r))(\delta(s))) .
\end{aligned}
$$

We set

$$
\begin{aligned}
\delta^{\prime} & =\lambda(s, w) \delta(s): \operatorname{Mref}(q+k) \rightarrow \underline{Q} \\
l^{\prime} & =\lambda(s, w) \lambda z l(w)(z) \in R^{\prime \operatorname{Mref}(q+k)} .
\end{aligned}
$$

Then $^{25} \delta^{\prime} \in$ paths $_{1}(Q)_{q+k}$ and $l^{\prime} \in \mathcal{M}(R)_{q+k}$ and therefore $\delta^{\prime} \triangleright l^{\prime} \in \mathcal{M}\left(Q \sim_{\mathrm{m}} R\right)_{q+k}$. We know that $g \circ \gamma \in$ paths $_{1}\left(Q \rightarrow_{\mathrm{m}} R\right)_{p}$ and hence $\lambda(r, s, w)\left(\delta^{\prime} \triangleright l^{\prime}\right)(s, w)(g(\gamma(r)))$ is measurable $\operatorname{Mref}(p+q+k) \rightarrow \mathbb{R}_{\geq 0}$. Now observe that

$\lambda(r, s, w)\left(\delta^{\prime} \triangleright l^{\prime}\right)(s, w)(g(\gamma(r)))=\lambda(r, s, w) l(w)(g(\gamma(r))(\delta(s)))$

so we have proven that $m \in \mathcal{M}(P \widehat{\otimes} Q)_{k}$. But remember that $\theta \in$ paths $_{1}(P \widehat{\otimes} Q)_{p_{0}}$, we have therefore $\lambda\left(r_{0}, w\right) m(w)\left(\theta\left(r_{0}\right)\right) \in$ $\operatorname{Meas}\left(\operatorname{Mref}\left(p_{0}+k\right), \mathbb{R}_{\geq 0}\right)$ and since $m(w)\left(\theta\left(r_{0}\right)\right)$ is nothing but $l(w)\left(f\left(\theta\left(r_{0}\right)\right)\right)$ we have $f \circ \theta \in$ paths $_{1}(R)_{p_{0}}$.

\subsection{Proof of Theorem 37}

Proof. Let $f \in \operatorname{CLin}_{\mathrm{m}}(\widehat{!} P, Q)$, the associated $g \in \operatorname{Cstab}(\underline{P}, \underline{Q})$ is defined by $g(x)=f\left(x^{\hat{!}}\right)$. Let $\gamma \in$ paths $_{1}(P)_{p}$, we have $g \circ \gamma=f \circ \gamma^{\hat{!}} \in$ paths $_{1}(Q)_{p}$ since $\gamma^{\hat{!}} \in$ paths $_{1}\left(\widehat{l}_{P}\right)_{p}$ and hence $g \in \operatorname{Cstab}(P, Q)$. Now let $g \in \operatorname{Cstab}_{\mathrm{m}}(P, Q)$ and let $f \in \mathrm{CLin}(\underline{\underline{P}}, \underline{Q})$ be the associated linear map, uniquely characterized by $\forall x \in \mathcal{B} P g(x)=f\left(x^{\grave{!}}\right)$. Let $\theta \in$ paths $_{1}(\widehat{!} P)_{p}$, we prove that $f \circ \theta \in$ paths $_{1}(Q)_{p}$ so let $m \in \mathcal{M}(Q)_{q}$, we define $l=\lambda s \lambda z m(s)(f(z)) \in \underline{?}^{\prime \operatorname{Mref}(q)}$ (linearity and continuity of $l(s)$ follows from those of $f)$. The fact that

\footnotetext{
${ }^{25}$ Because $\mathbf{M}$ is cartesian and measurability tests and paths are closed under precomposition by morphisms of $\mathbf{M}$.
}

$\lambda s l(s)(z) \in \operatorname{Meas}\left(\operatorname{Mref}(q), \mathbb{R}_{\geq 0}\right)$ for each $z \in \underline{\underline{P}}$ follows from $m \in \mathcal{M}(Q)_{q}$. Let $\gamma \in$ paths $_{1}(P)_{p_{0}}$, we have

$$
\begin{aligned}
\lambda\left(r_{0}, s\right) l(s)\left(\gamma\left(r_{0}\right)^{\dagger}\right) & =\lambda\left(r_{0}, s\right) m(s)\left(g\left(\gamma\left(r_{0}\right)\right)\right. \\
& \in \operatorname{Meas}\left(\operatorname{Mref}\left(r_{0}+s\right), \mathbb{R}_{\geq 0}\right)
\end{aligned}
$$

since $g$ is measurable, hence $m \in \mathcal{M}(\widehat{!} P)$. Since $\theta \in$ paths $_{1}(\widehat{!} P)_{p}$ it follows that $\lambda(r, s) l(s)(\theta(r)) \in \operatorname{Meas}\left(\operatorname{Mref}(r+s), \mathbb{R}_{\geq 0}\right)$ but $l(s)(\theta(r))=m(s)(f(\theta(r)))$ and so we have proven that $f \circ \theta \in$ paths $_{1}(Q)_{p}$.

\subsection{The pentagon}

We have to prove commutation of the external pentagon of Figure 1 where the morphisms $\beta_{i}, \gamma_{i}$ are instances ${ }^{26}$ of $\widetilde{\alpha}, \alpha_{i}$ are obtained by applying $\overline{\mathrm{P}}$ to $\alpha$ and $\pi_{i}$ are instances of $\pi$, that is $\beta_{2} \beta_{1}=\beta_{5} \beta_{4} \beta_{3}$. This is reduced to the commutation of the internal pentagon involving $\alpha_{1}, \ldots, \alpha_{5}$ by observing that

$$
\beta_{2} \beta_{1}=\left(\pi_{11} \pi_{10} \pi_{9}\right)^{-1} \alpha_{2} \alpha_{1}\left(\pi_{3} \pi_{2} \pi_{1}\right)
$$

that is $\pi_{11} \pi_{10} \pi_{9} \beta_{2} \beta_{1}=\alpha_{2} \alpha_{1}\left(\pi_{3} \pi_{2} \pi_{1}\right)$ and similarly for $\beta_{5} \beta_{4} \beta_{3}$. This is done by pasting five kinds of commutative squares of which we give examples, explaining why they commute.

- The diagram involving $\beta_{1}, \pi_{4}, \pi_{1}$ and $\gamma_{1}$ which commutes by naturality of $\widetilde{\alpha}$.

- The diagram involving $\pi_{4}, \pi_{6}, \pi_{5}$ and $\pi_{7}$ which commutes by functoriality of $\widehat{\otimes}$.

- The diagram involving $\gamma_{1}, \pi_{6}, \pi_{8}, \pi_{2}, \pi_{3}$ and $\alpha_{1}$ whose commutation results from the definition of $\alpha^{0}$ and $\widetilde{\alpha}$.

- The diagram involving $\beta_{3}, \pi_{13}, \pi_{14}, \pi_{1}, \pi_{2}$ and $\alpha_{6}$ whose commutation results from the definition of $\alpha^{0}$ and $\widetilde{\alpha}$.

- The diagram involving $\alpha_{6}, \pi_{12}, \pi_{3}$ and $\alpha_{3}$ which results from the naturality of $\pi$.

\footnotetext{
${ }^{26}$ Possibly involving tensorisations with identities, the same for the next uses of the word "instance".
} 
$\left(\left(\overline{\mathrm{P}} X_{1} \widehat{\otimes} \overline{\mathrm{P}} X_{2}\right) \widehat{\otimes} \overline{\mathrm{P}} X_{3}\right) \widehat{\otimes} \overline{\mathrm{P}} X_{4} \longrightarrow\left(\overline{\mathrm{P}} X_{1} \widehat{\otimes} \overline{\mathrm{P}} X_{2}\right) \widehat{\otimes}\left(\overline{\mathrm{P}} X_{3} \widehat{\otimes} \overline{\mathrm{P}} X_{4}\right)$

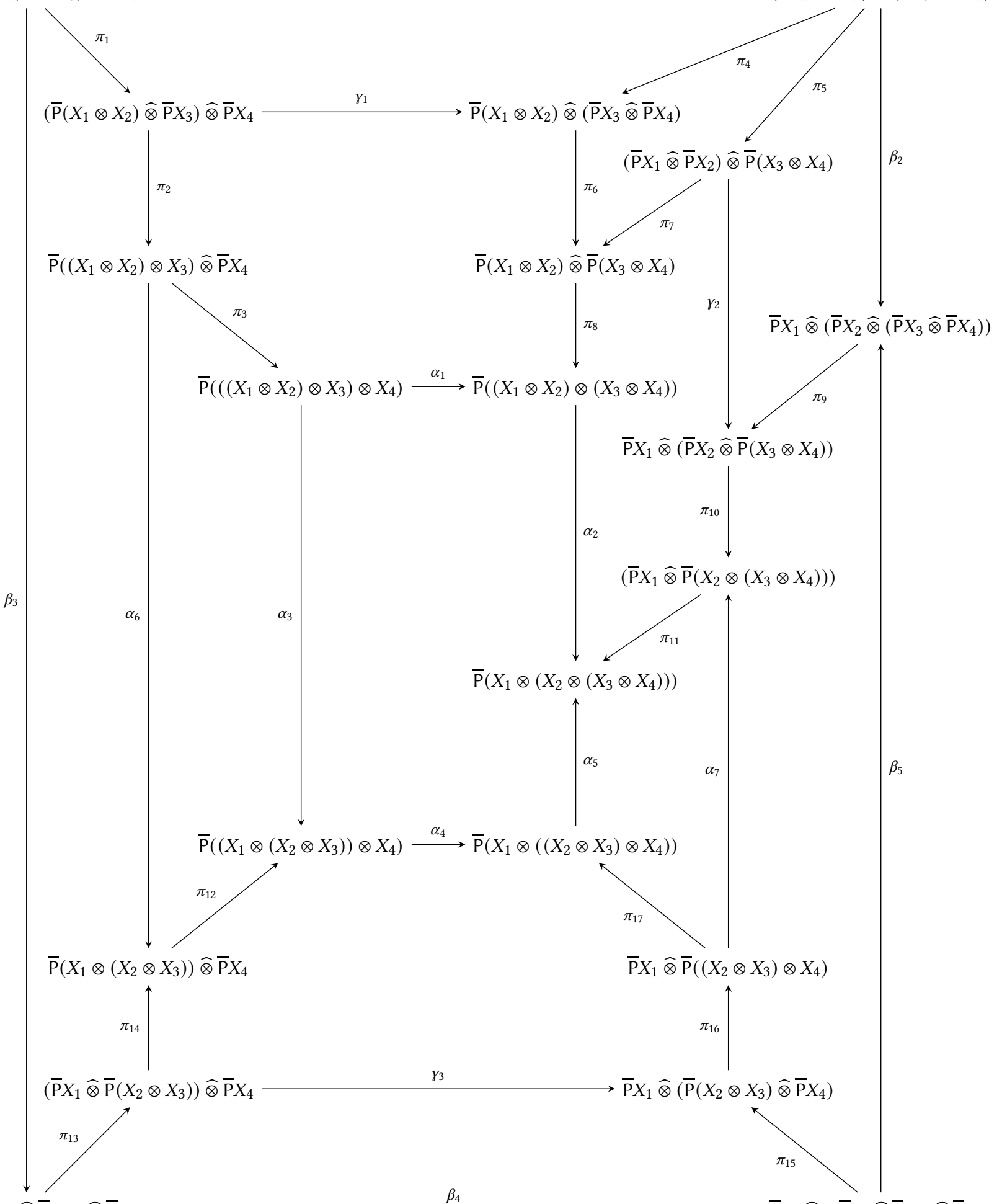

$\left(\overline{\mathrm{P}} X_{1} \widehat{\otimes}\left(\overline{\mathrm{P}} X_{2} \widehat{\otimes} \overline{\mathrm{P}} X_{3}\right)\right) \widehat{\otimes} \overline{\mathrm{P}} X_{4}$

$\longrightarrow \overline{\mathrm{P}} X_{1} \widehat{\otimes}\left(\left(\overline{\mathrm{P}} X_{2} \widehat{\otimes} \overline{\mathrm{P}} X_{3}\right) \widehat{\otimes} \overline{\mathrm{P}} X_{4}\right)$

Figure 1. Pentagon diagram 